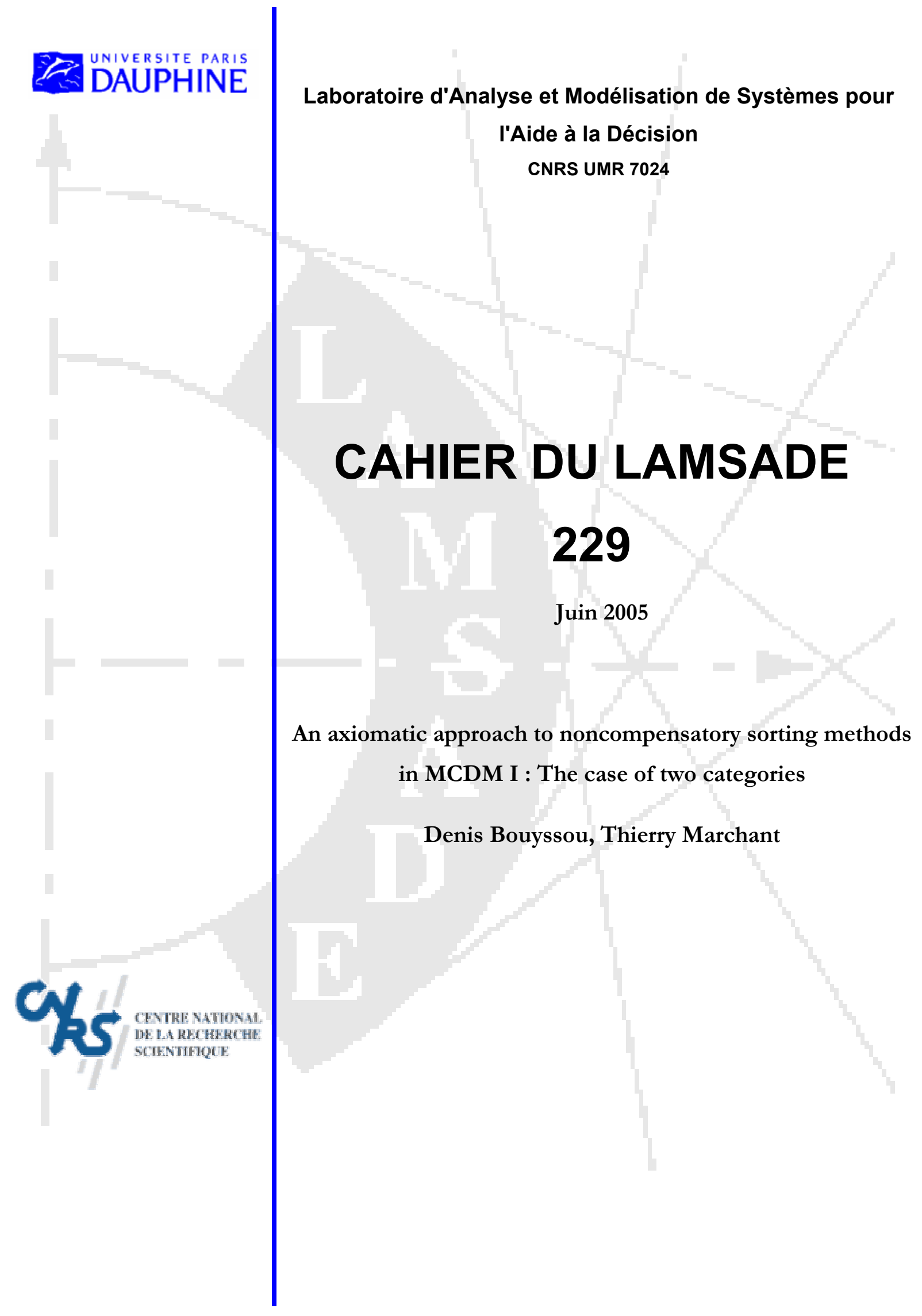




\section{An axiomatic approach to noncompensatory sorting methods in MCDM, I: The case of two categories ${ }^{1}$}

\author{
Denis Bouyssou ${ }^{2}$ \\ CNRS - LAMSADE
}

\author{
Thierry Marchant ${ }^{3}$ \\ Ghent University
}

10 January 2005

Revised 25 October 2005

\footnotetext{
${ }^{1}$ We wish to thank Jose Figueira and Marc Pirlot for their helpful comments on an earlier draft of this text. Our greatest debt is to Salvatore Greco, Benedetto Matarazzo and Roman Słowiński who alerted us on the relation between our results on noncompensatory sorting models and the results in Słowiński et al. (2002) on sorting models using a Sugeno integral. Furthermore, their detailed comments have much contributed to improve an earlier version of this text. The usual caveat clearly applies.

${ }^{2}$ LAMSADE, Université Paris Dauphine, Place du Maréchal de Lattre de Tassigny, F-75775 Paris Cedex 16, France, tel: +331440548 98, fax: +3314405 40 91, e-mail: bouyssou@lamsade.dauphine.fr, Corresponding author.

${ }^{3}$ Ghent University, Department of Data Analysis, H. Dunantlaan 1, B9000 Gent, Belgium, tel: +32 926463 73, fax: +32 926464 87, e-mail: thierry.marchant@UGent. be.
} 


\begin{abstract}
In the literature on MCDM, many methods have been proposed in order to sort alternatives evaluated on several attributes into ordered categories. Most of them were proposed on an ad hoc basis. The purpose of this paper is to contribute to a recent trend of research aiming at giving these methods sound theoretical foundations. Using tools from conjoint measurement, we provide an axiomatic analysis of the partitions of alternatives into two categories that can be obtained using what we call "noncompensatory sorting models". These models have strong links with the pessimistic version of ELECTRE TRI. Our analysis allows to pinpoint what appears to be the main distinctive features of ELECTRE TRI when compared to other sorting methods. It also gives hints on the various methods that have been proposed to assess the parameters of ELECTRE TRI on the basis of assignment examples.
\end{abstract}

Keywords: Decision with multiple attributes, Sorting, Conjoint measurement, Noncompensatory sorting methods, ELECTRE TRI. 


\section{Contents}

1 Introduction and motivation 1

2 Definitions and notation 3

2.1 Binary relations and partitions . . . . . . . . . . . 3

2.2 The setting . . . . . . . . . . . . . . . . 4

3 A general measurement framework 4

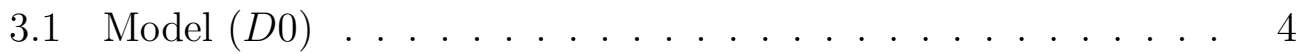

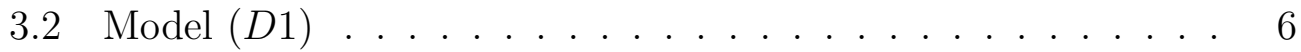

3.3 Interpretations of model $(D 1) \ldots \ldots . \ldots 9$

4 ELECTRE TRI 13

5 The noncompensatory sorting model 14

5.1 Definitions . . . . . . . . . . . . . . . 14

5.2 Particular cases . . . . . . . . . . . . . . . 17

5.3 Axioms and results . . . . . . . . . . . . . . . 18

5.4 The noncompensatory sorting model and the Sugeno integral . 22

5.5 Extensions . . . . . . . . . . . . . . . . 24

5.6 Uniqueness and degenerate attributes . . . . . . . . . . . . 28

5.7 Conjunctive and disjunctive sorting models . . . . . . . . . 30

6 The noncompensatory sorting model with veto 30

6.1 Definitions ...................... 30

6.2 Axioms and results ................. . . . . 31

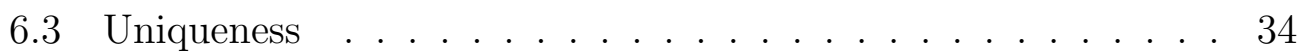

6.4 Extensions . . . . . . . . . . . . . . . . . 36

$\begin{array}{lll}7 & \text { Discussion } & 40\end{array}$

$\begin{array}{ll}\text { References } & 42\end{array}$ 


\section{Introduction and motivation}

MCDM has traditionally been concerned with decision situations in which the objective is either to select an alternative from a set of alternatives evaluated on several attributes or to rank order this set (see, e.g., Belton and Stewart, 2001; Bouyssou et al., 2000). In such situations, the usual practice is to build a recommendation on the basis of a binary relation comparing alternatives in terms of preference. In such an approach, the recommendation is based on a relative evaluation model of the alternatives as given by the preference relation. Since these techniques manipulate binary relations on product sets, it should be no surprise that conjoint measurement (see Krantz et al., 1971; Wakker, 1989) has proved to be quite a powerful tool to analyze and compare them. It has also been an inspiring guide to many assessment techniques (see Keeney and Raiffa, 1976; von Winterfeldt and Edwards, 1986).

The use of relative evaluation models is not always appropriate to build meaningful recommendations. Indeed the "best" alternatives, while being superior to all others, may well not be desirable. When such elements appear important, one may turn to evaluation models having a more absolute character. This leads to comparing alternatives not between them but to pre-defined norms. The result of such an analysis is a partition of the set of alternatives into several categories defined with respect to these norms. This is what Roy (1996) called the sorting problem formulation. This is the subject of this paper.

Sorting problems come in two rather different guises depending on whether the categories used to sort the alternatives are ordered in terms of desirability (for very good introductions to the subject, see Greco et al., 1999, 2002a,b; Zopounidis and Doumpos, 2000b, 2002). The situation in which they are not, usually called "classification", is common in pattern recognition or medical diagnosis. Such problems have been studied rather intensively in Statistics, Operations Research and Artificial Intelligence (see Hand, 1981; Pawlak, 1991; Pawlak and Słowiński, 1994; Weiss and Kulikowski, 1991). The situation in which the categories are ordered is also quite common. It occurs, for instance, when a credit analyst rates credit applications or when an academic program is enrolling students. It has recently attracted much attention in the literature on MCDM (see Greco et al., 1999, 2002a, 2005; Zopounidis and Doumpos, 2000b, 2002, for reviews). Several methods have been designed to tackle such problems such as UTADIS (see Jacquet-Lagrèze, 1995; Zopounidis and Doumpos, 2000c), ELECTRE TRI (see Mousseau et al., 2000b; Roy and Bouyssou, 1993; Wei, 1992), filtering methods (see Henriet, 2000; Perny, 1998), methods based on the Choquet integral (see Marichal et al., 2005; Marichal and Roubens, 2001; Meyer and Roubens, 2005), methods inspired 
by PROMETHEE (Doumpos and Zopounidis, 2002, 2004; Figueira et al., 2004) or the interactive approach introduced in Köksalan and Ulu (2003). Most of these sorting techniques were proposed on a more or less ad hoc basis.

The aim of this paper is to contribute to a recent trend of research (see Greco et al., 2001b; Słowiński et al., 2002) aiming at providing sound theoretical foundations to such methods. More precisely, we propose an axiomatic analysis of what we shall call "noncompensatory sorting models". The main characteristic of these models is that they rely on rather poor information on each attribute. These models have close connections with ELECTRE TRI and our analysis may be considered as an attempt to provide a firm axiomatic basis to this particular sorting technique. The choice of the ELECTRE TRI method was motivated by the fact that it has attracted much attention in the literature. It will turn out that some of our results have close connections with the ones proposed in Greco et al. (2001b) and Słowiński et al. (2002).

This paper concentrates on sorting problems with two categories. Besides being much simpler than the general case, this situation deserves special attention since it tends to blur the distinction between the case of ordered and unordered categories. A companion paper (Bouyssou and Marchant, 2005) generalizes our results to the case of an arbitrary (finite) number of categories; in this case, the distinction between the situations with ordered and unordered categories is crucial.

Technically, our strategy will be to use conjoint measurement techniques to deal with partitions, instead of binary relations, defined on Cartesian products. This strategy was first proposed by Goldstein (1991) in the context of categorization tasks in Psychology. It was independently rediscovered and much developed in Greco et al. (2001b) and Słowiński et al. (2002). Recently, Nakamura (2004) has explored a related path for decision making under risk.

The rest of this paper is organized as follows. We introduce our setting in Section 2. Section 3 introduces the general measurement framework within which we shall work. Section 4 recalls the principles of the ELECTRE TRI technique. Section 5 deals with the case of noncompensatory sorting models. These models are roughly equivalent to the ELECTRE TRI method when there is no discordance effect. Section 6 extends this analysis to include the possibility of veto effects as in ELECTRE TRI. A final section discusses our findings and presents directions for future research.

Throughout the paper, remarks contain comments that can be skipped on first reading without loss of continuity. 


\section{Definitions and notation}

\subsection{Binary relations and partitions}

We use a standard vocabulary for binary relations. An equivalence (resp. a weak order; a total order; a semiorder) is a reflexive, symmetric and transitive (resp. complete and transitive; complete, antisymmetric and transitive; complete, Ferrers and semi-transitive) relation.

When $T$ is an equivalence relation on $A, A / T$ will denote the set of equivalence classes of $T$ on $A$. A partition of $A$ is a collection of nonempty subsets of $A$ that are pairwise disjoint and such that the union of the elements in this collection is $A$. It is clear that, when $T$ is an equivalence relation on $A, A / T$ is a partition of $A$. Indeed, defining a partition of $A$ is tantamount to defining an equivalence relation on $A$.

When $T$ is reflexive and transitive, its symmetric part $\iota(T)$ is an equivalence. It will prove convenient to speak of the equivalence classes of $T$ to mean the equivalence classes of its symmetric part $\iota(T)$. When $T$ is a weak order, it induces on a total order on $A / \iota(T)$. When $T$ is a weak order and $A / \iota(T)$ is finite, we shall often speak of the first or last equivalence class of $T$.

Let $T$ be a binary relation on $A$. It is well-known that there is a realvalued function $f$ on $A$ such that, for all $a, b \in A$,

$$
a T b \Leftrightarrow f(a)=f(b),
$$

if and only if $T$ is an equivalence and there is a one-to-one correspondence between $A / T$ and some subset of $\mathbb{R}$.

Let $T$ be a weak order on $A$. Following, e.g., Krantz et al. (1971, Chapter 2), we say that $B$ is dense in $A$ for $T$ if, for all $a, b \in A,[a T b$ and $\operatorname{Not}[b T a]] \Rightarrow[a T c$ and $c T b$, for some $c \in B]$. It is well-known (Fishburn, 1970; Krantz et al., 1971) that there is a real-valued function $f$ on $A$ such that, for all $a, b \in A$,

$$
a T b \Leftrightarrow f(a) \geq f(b)
$$

if and only if $T$ is a weak order and there is a finite or countably infinite set $B \subseteq A$ that is dense in $A$ for $T$.

Let $T$ and $T^{\prime}$ be two weak orders on $A$. We say that $T^{\prime}$ refines $T$ if, for all $a, b \in A, a T^{\prime} b \Rightarrow a T b$. If $T^{\prime}$ refines $T$ and there is a set $B$ that is dense in $A$ for $T^{\prime}, B$ is also dense in $A$ for $T$. 


\section{$2.2 \quad$ The setting}

Let $n \geq 2$ be an integer and $X=X_{1} \times X_{2} \times \cdots \times X_{n}$ be a set of objects. Elements $x, y, z, \ldots$ of $X$ will be interpreted as alternatives evaluated on a set $N=\{1,2, \ldots, n\}$ of attributes. For any nonempty subset $J$ of the set of attributes $N$, we denote by $X_{J}\left(\operatorname{resp} . X_{-J}\right)$ the set $\prod_{i \in J} X_{i}\left(\operatorname{resp} . \prod_{i \notin J} X_{i}\right)$. With customary abuse of notation, $\left(x_{J}, y_{-J}\right)$ will denote the element $w \in X$ such that $w_{i}=x_{i}$ if $i \in J$ and $w_{i}=y_{i}$ otherwise. When $J=\{i\}$ we shall simply write $X_{-i}$ and $\left(x_{i}, y_{-i}\right)$.

Our primitives consist in a twofold partition $\langle\mathscr{A}, \mathscr{U}\rangle$ of the set $X$; this means that the sets $\mathscr{A}$ and $\mathscr{U}$ are nonempty and disjoint and that their union is the entire set $X$. Our central aim is to study various models allowing to represent the information contained in $\langle\mathscr{A}, \mathscr{U}\rangle$. We interpret the partition $\langle\mathscr{A}, \mathscr{U}\rangle$ as the result of a sorting model applied to the alternatives in $X$. Although the ordering of the categories is not part of our primitives, it is useful to interpret the set $\mathscr{A}$ as containing sAtisfactory objects, while $\mathscr{U}$ contains Unsatisfactory ones.

We say that an attribute $i \in N$ is influent for $\langle\mathscr{A}, \mathscr{U}\rangle$ if there are $x_{i}, y_{i} \in$ $X_{i}$ and $a_{-i} \in X_{-i}$ such that $\left(x_{i}, a_{-i}\right) \in \mathscr{A}$ and $\left(y_{i}, a_{-i}\right) \in \mathscr{U}$. We say that an attribute is degenerate if it is not influent. Note that the fact that $\langle\mathscr{A}, \mathscr{U}\rangle$ is a partition implies that there is at least one influent attribute in $N$. A degenerate attribute has no influence whatsoever on the sorting of the alternatives and may be suppressed from $N$. However, because our analysis of twofold partitions is intended to be used to tackle the case of general partitions, we do not suppose below that degenerate attributes have been suppressed from $N$.

\section{A general measurement framework}

Goldstein (1991) suggested the use of conjoint measurement techniques for the analysis of twofold and threefold partitions of a set of multi-attributed alternatives. His analysis was independently rediscovered and much developed in Greco et al. (2001b) and Słowiński et al. (2002). We briefly recall here the main points of the analysis in the above papers for the case of twofold partitions.

\subsection{Model $(D 0)$}

Consider first a measurement model in which, for all $x \in X$,

$$
x \in \mathscr{A} \Leftrightarrow F\left(u_{1}\left(x_{1}\right), u_{2}\left(x_{2}\right), \ldots, u_{n}\left(x_{n}\right)\right)>0
$$


where $u_{i}$ is a real-valued function on $X_{i}$ and $F$ is a real-valued function on $\prod_{i=1}^{n} u_{i}\left(X_{i}\right)$ that is one-to-one in each variable (i.e., $F\left(\alpha_{1}, \ldots, \alpha_{i-1}, \beta_{i}\right.$, $\left.\left.\alpha_{i+1}, \ldots, \alpha_{n}\right)=F\left(\alpha_{1}, \ldots, \alpha_{i-1}, \gamma_{i}, \alpha_{i+1}, \ldots, \alpha_{n}\right) \Rightarrow \beta_{i}=\gamma_{i}\right)$.

Define on each $X_{i}$ a binary relation $\sim_{i}$ letting, for all $x_{i}, y_{i} \in X_{i}$,

$$
x_{i} \sim_{i} y_{i} \Leftrightarrow\left[\text { for all } a_{-i} \in X_{-i},\left(y_{i}, a_{-i}\right) \in \mathscr{A} \Leftrightarrow\left(x_{i}, a_{-i}\right) \in \mathscr{A}\right] .
$$

It is clear that $\sim_{i}$ is an equivalence, being reflexive, symmetric and transitive. Note that, when attribute $i \in N$ is influent, $\sim_{i}$ will have at least two distinct equivalence classes. We have:

\section{Lemma 1}

For all $x, y \in X$,

1. $\left[y \in \mathscr{A}\right.$ and $\left.x_{i} \sim_{i} y_{i}\right] \Rightarrow\left(x_{i}, y_{-i}\right) \in \mathscr{A}$,

2. $\left[x_{i} \sim_{i} y_{i}\right.$, for all $\left.i \in N\right] \Rightarrow[x \in \mathscr{A} \Leftrightarrow y \in \mathscr{A}]$.

PROOF

Part 1 follows from the definition of $\sim_{i}$. Part 2 easily follows from Part 1.

The following proposition is due to Goldstein (1991, Theorem 1, Part 1).

\section{Proposition 2}

Let $\langle\mathscr{A}, \mathscr{U}\rangle$ be a twofold partition of a set $X$. Then $\langle\mathscr{A}, \mathscr{U}\rangle$ has a representation in model (DO) if and only if, for all $i \in N$, there is a one-to-one correspondence between $X_{i} / \sim_{i}$ and some subset of $\mathbb{R}$.

PROOF

Necessity. Suppose that $\langle\mathscr{A}, \mathscr{U}\rangle$ has a representation in model $(D 0)$. If $\operatorname{Not}\left[x_{i} \sim_{i} y_{i}\right]$, we must have $u_{i}\left(x_{i}\right) \neq u_{i}\left(y_{i}\right)$. Therefore $X_{i} / \sim_{i}$ is a lower bound on the cardinality of the image of $X_{i}$ by $u_{i}$. Since $u_{i}$ is real-valued, this implies that there must exist a one-to-one correspondence between $X_{i} / \sim_{i}$ and some subset of $\mathbb{R}$.

Sufficiency. By hypothesis, there is a one-to-one correspondence between $X_{i} / \sim_{i}$ and some subset of $\mathbb{R}$. This implies that there is a real-valued function $u_{i}$ on $X_{i}$ such that, for all $x_{i}, y_{i} \in X_{i}$,

$$
x_{i} \sim_{i} y_{i} \Leftrightarrow u_{i}\left(x_{i}\right)=u_{i}\left(y_{i}\right) .
$$

Consider, on each $i \in N$, any function $u_{i}$ satisfying (1). Define $F$ on $\prod_{i=1}^{n} u_{i}\left(X_{i}\right)$ letting:

$$
F\left(u_{1}\left(x_{1}\right), u_{2}\left(x_{2}\right), \ldots, u_{n}\left(x_{n}\right)\right)=\left\{\begin{array}{l}
\exp \left(\sum_{i=1}^{n} u_{i}\left(x_{i}\right)\right) \text { if } x \in \mathscr{A} \\
-\exp \left(-\sum_{i=1}^{n} u_{i}\left(x_{i}\right)\right) \text { otherwise }
\end{array}\right.
$$

The well-definedness of $F$ follows from Part 2 of lemma 1 . That $F$ is one-toone in each variable follows from its definition. 
As soon as the cardinality of each set $X_{i}$ is not "too large" (e.g., when each $X_{i}$ has at most the cardinality of $\mathbb{R}$ ), this shows that all twofold partitions can be represented in model $(D 0)$. Notice that if $F$ is not supposed to be oneto-one in each variable, it is still true that $\operatorname{Not}\left[x_{i} \sim_{i} y_{i}\right] \Rightarrow u_{i}\left(x_{i}\right) \neq u_{i}\left(y_{i}\right)$. This shows that this model is equivalent to model $(D 0)$.

\section{Remark 3}

The uniqueness of the representation of $\langle\mathscr{A}, \mathscr{U}\rangle$ in model $(D 0)$ is very weak. As this point was not detailed in Goldstein (1991), we briefly analyze it below. Independently on each $i \in N$, it is not difficult to see that any function $u_{i}$ on $X_{i}$ such that, for all $x_{i}, y_{i} \in X_{i}$,

$$
\operatorname{Not}\left[x_{i} \sim_{i} y_{i}\right] \Rightarrow u_{i}\left(x_{i}\right) \neq u_{i}\left(y_{i}\right),
$$

may be used instead of a function $u_{i}$ satisfying (1). Take on each $i \in N$ any functions $u_{i}$ satisfying (3). Any function $g$ from $\prod_{i=1}^{n} u_{i}\left(X_{i}\right)$ into $(0,+\infty)$ that is one-to-one in each variable and any function $h$ from $\prod_{i=1}^{n} u_{i}\left(X_{i}\right)$ into $(-\infty, 0]$ that is one-to-one in each variable may be used in order to define $F$ letting:

$$
F\left(\left[u_{i}\left(x_{i}\right)\right]\right)= \begin{cases}g\left(\left[u_{i}\left(x_{i}\right)\right]\right) & \text { if } x \in \mathscr{A}, \\ h\left(\left[u_{i}\left(x_{i}\right)\right]\right) & \text { otherwise }\end{cases}
$$

It is furthermore clear that only such functions may be used so that the combination of equations (3) and (4) describe the set of all numerical representations of $\langle\mathscr{A}, \mathscr{U}\rangle$ in model $(D 0)$.

\section{Remark 4}

Notice that the roles of $\mathscr{A}$ and $\mathscr{U}$ are entirely symmetric in model $(D 0)$. Indeed, the proof of Proposition 2 shows that $F$ may always be chosen to be non-null. Therefore, we can re-write model $(D 0)$ as follows:

$$
\begin{aligned}
& x \in \mathscr{A} \Leftrightarrow F\left(u_{1}\left(x_{1}\right), u_{2}\left(x_{2}\right), \ldots, u_{n}\left(x_{n}\right)\right)>0, \\
& x \in \mathscr{U} \Leftrightarrow F\left(u_{1}\left(x_{1}\right), u_{2}\left(x_{2}\right), \ldots, u_{n}\left(x_{n}\right)\right)<0 .
\end{aligned}
$$

Taking $G=-F$ shows the symmetry of the roles $\mathscr{A}$ and $\mathscr{U}$.

\subsection{Model $(D 1)$}

Consider the variant of model $(D 0)$ in which $F$ is supposed to be increasing (resp. nondecreasing) in each variable. This defines model $(D 1)$ (resp. $(D 2)$ ). Model $(D 1)$ contains as a particular case the additive model for sorting in which, for all $x \in X$,

$$
x \in \mathscr{A} \Leftrightarrow \sum_{i=1}^{n} u_{i}\left(x_{i}\right)>0,
$$


that is at the heart of the UTADIS technique (Jacquet-Lagrèze, 1995; Zopounidis and Doumpos, 2000c) and its variants (Zopounidis and Doumpos, 2000a). We shall see below that models (D1) and (D2) are equivalent and that they have close links with the ELECTRE TRI technique.

In order to analyze models $(D 1)$ and $(D 2)$, we define on each $X_{i}$ the binary relation $\succsim_{i}$ letting, for all $x_{i}, y_{i} \in X_{i}$,

$$
x_{i} \succsim_{i} y_{i} \Leftrightarrow\left[\text { for all } a_{-i} \in X_{-i},\left(y_{i}, a_{-i}\right) \in \mathscr{A} \Rightarrow\left(x_{i}, a_{-i}\right) \in \mathscr{A}\right] .
$$

It is not difficult to see that, by construction, $\succsim_{i}$ is reflexive and transitive. We denote by $\succ_{i}\left(\right.$ resp. $\left.\sim_{i}\right)$ the asymmetric (resp. symmetric) part of $\succsim_{i}$ (hence, the relation $\sim_{i}$ coincides with the one used above).

We say that the partition $\langle\mathscr{A}, \mathscr{U}\rangle$ is linear on attribute $i \in N$ (condition linear $_{i}$ ) if, for all $x_{i}, y_{i} \in X_{i}$ and all $a_{-i}, b_{-i} \in X_{-i}$,

$$
\left.\begin{array}{l}
\left(x_{i}, a_{-i}\right) \in \mathscr{A} \\
\text { and } \\
\left(y_{i}, b_{-i}\right) \in \mathscr{A}
\end{array}\right\} \Rightarrow\left\{\begin{array}{c}
\left(y_{i}, a_{-i}\right) \in \mathscr{A} \\
\text { or } \\
\left(x_{i}, b_{-i}\right) \in \mathscr{A}
\end{array}\right.
$$

The partition is said to be linear if it is linear on all $i \in N$. This condition was first proposed in Goldstein (1991) and independently rediscovered (and generalized) in Greco et al. (2001b) and Słowiński et al. (2002). The adaptation of this condition to the study of binary relations, adaptation first suggested by Goldstein (1991), is central in the analysis of the nontransitive decomposable models analyzed in Bouyssou and Pirlot (1999, 2002b, 2004a).

The following lemma takes note of the consequences of condition linear $_{i}$ on the relation $\succsim_{i}$ and shows that linearity is necessary for model $(D 1)$.

\section{Lemma 5}

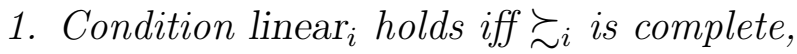

2. If $\langle\mathscr{A}, \mathscr{U}\rangle$ has a representation in model (D2) then it is linear.

PROOF

Part 1. We have $\operatorname{Not}\left[\right.$ linear $\left._{i}\right]$ if and only if, for some $x_{i}, y_{i} \in X_{i}$ and some $a_{-i}, b_{-i} \in X_{-i},\left(x_{i}, a_{-i}\right) \in \mathscr{A},\left(y_{i}, b_{-i}\right) \in \mathscr{A},\left(x_{i}, b_{-i}\right) \notin \mathscr{A}$ and $\left(y_{i}, a_{-i}\right) \notin \mathscr{A}$. This is equivalent to saying that $\succsim_{i}$ is not complete.

Part 2. Suppose that $\left(x_{i}, a_{-i}\right) \in \mathscr{A}$ and $\left(y_{i}, b_{-i}\right) \in \mathscr{A}$. This implies, using model (D2) and abusing notation in an obvious way, $F\left(u_{i}\left(x_{i}\right),\left[u_{j}\left(a_{j}\right)\right]_{j \neq i}\right)>0$ and $F\left(u_{i}\left(y_{i}\right),\left[u_{j}\left(b_{j}\right)\right]_{j \neq i}\right)>0$. If $u_{i}\left(x_{i}\right) \geq u_{i}\left(y_{i}\right)$ the latter equation implies, using the nondecreasingness of $F, F\left(u_{i}\left(x_{i}\right),\left[u_{j}\left(b_{j}\right)\right]_{j \neq i}\right)>0$ so that $\left(x_{i}, b_{-i}\right) \in \mathscr{A}$. If $u_{i}\left(y_{i}\right)>u_{i}\left(x_{i}\right)$, the former equation implies, using the nondecreasingness of $F, F\left(u_{i}\left(y_{i}\right),\left[u_{j}\left(a_{j}\right)\right]_{j \neq i}\right)>0$ so that $\left(y_{i}, a_{-i}\right) \in \mathscr{A}$. Hence, linear $_{i}$ holds, for all $i \in N$. 
The following proposition is due to Goldstein (1991, Theorem 2) and to Greco et al. (2001b, Theorem 2.1, Part 2).

\section{Proposition 6}

Let $\langle\mathscr{A}, \mathscr{U}\rangle$ be a twofold partition of a set $X$. There is a representation of $\langle\mathscr{A}, \mathscr{U}\rangle$ in model (D1) if and only if it is linear and, for all $i \in N$, there is a finite or countably infinite set $X_{i}^{\prime} \subseteq X_{i}$ that is dense in $X_{i}$ for $\succsim_{i}$.

Furthermore:

- if $\langle\mathscr{A}, \mathscr{U}\rangle$ has a representation in model (D1), it has a representation in which, for all $i \in N, u_{i}$ is a numerical representation of $\succsim_{i}$,

- models (D1) and model (D2) are equivalent,

\section{ProOF}

Let us show that the conditions are necessary for model $(D 2)$. The necessity of linearity results from Part 2 of lemma 5 . Observe that if $\langle\mathscr{A}, \mathscr{U}\rangle$ has a representation in model $(D 2)$, we must have:

$$
x_{i} \succ_{i} y_{i} \Rightarrow u_{i}\left(x_{i}\right)>u_{i}\left(y_{i}\right) .
$$

Hence, the weak order induced on $X_{i}$ by $u_{i}$ refines $\succsim_{i}$. It follows that there must be a finite or countably infinite set $X_{i}^{\prime} \subseteq X_{i}$ that is dense in $X_{i}$ for $\succsim_{i}$.

Let us now show that the conditions imply model $(D 1)$, which will also show that models $(D 1)$ and $(D 2)$ are equivalent. Part 1 of lemma 5 implies that $\succsim_{i}$ is a weak order. Since there is a finite or countably infinite set $X_{i}^{\prime} \subseteq X_{i}$ that is dense in $X_{i}$ for $\succsim_{i}$, there is a real-valued function $u_{i}$ on $X_{i}$ such that, for all $x_{i}, y_{i} \in X_{i}$ :

$$
x_{i} \succsim_{i} y_{i} \Leftrightarrow u_{i}\left(x_{i}\right) \geq u_{i}\left(y_{i}\right) .
$$

Consider, on each $i \in N$ any function $u_{i}$ satisfying (6). Define $F$ on $\prod_{i=1}^{n} u_{i}\left(X_{i}\right)$ using (2). The well-definedness of $F$ follows from Part 1 of lemma 1. The increasingness of $F$ follows from its definition, (6) and the definition of $\succsim_{i}$.

In view of the equivalence between models $(D 1)$ and $(D 2)$, we concentrate below on model $(D 1)$.

\section{Remark 7}

The uniqueness of the representation of $\langle\mathscr{A}, \mathscr{U}\rangle$ in model $(D 1)$ is quite weak. Independently on any attribute, the only constraint on $u_{i}$ is that (5) holds. Using any such functions $u_{i}$, any function $g$ from $\prod_{i=1}^{n} u_{i}\left(X_{i}\right)$ into $(0,+\infty)$ that is increasing in all its arguments and any function $h$ from 
$\prod_{i=1}^{n} u_{i}\left(X_{i}\right)$ into $(-\infty, 0]$ that is increasing in all its arguments (when restricted to $\left.\prod_{i=1}^{n} u_{i}\left(X_{i}\right)\right)$ may be used in order to define $F$ letting:

$$
F\left(\left[u_{i}\left(x_{i}\right)\right]\right)= \begin{cases}g\left(\left[u_{i}\left(x_{i}\right)\right]\right) & \text { if } x \in \mathscr{A}, \\ h\left(\left[u_{i}\left(x_{i}\right)\right]\right) & \text { otherwise. }\end{cases}
$$

It is furthermore clear that only such functions may be used so that the combination of equations (5) and (7) describe the set of all numerical representations of $\langle\mathscr{A}, \mathscr{U}\rangle$ in model $(D 1)$.

The uniqueness of the representation in model $(D 2)$ is even weaker. It can easily be analyzed along similar lines. Let us simply note here that, if $\langle\mathscr{A}, \mathscr{U}\rangle$ has a representation in model $(D 2)$, it also has a representation in which $F\left(\left[u_{i}\left(x_{i}\right)\right]\right)=\alpha>0$, for all $x \in \mathscr{A}$ and $F\left(\left[u_{i}\left(x_{i}\right)\right]\right)=\beta<0$, for all $x \in \mathscr{U}$. In this case $F$ also represents the equivalence relation that is naturally associated with $\langle\mathscr{A}, \mathscr{U}\rangle$.

\section{Remark 8}

The roles of $\mathscr{A}$ and $\mathscr{U}$ are entirely symmetric in model $(D 1)$. Indeed, instead of using $\succsim_{i}$, we could have based the analysis on the relation $\succsim_{i}^{\prime}$ defined by:

$$
x_{i} \succsim_{i}^{\prime} y_{i} \Leftrightarrow\left[\text { for all } a_{-i} \in X_{-i},\left(y_{i}, a_{-i}\right) \in \mathscr{U} \Rightarrow\left(x_{i}, a_{-i}\right) \in \mathscr{U}\right] .
$$

In this case, we would have obtained a model in which:

$$
x \in \mathscr{U} \Leftrightarrow G\left(v_{1}\left(x_{1}\right), v_{2}\left(x_{2}\right), \ldots, v_{n}\left(x_{n}\right)\right)>0,
$$

with $G$ increasing in all its arguments.

Another way to notice this symmetry is to observe that the condition obtained replacing $\mathscr{A}$ by $\mathscr{U}$ in the expression of linear ${ }_{i}$ gives a condition that is equivalent to linear ${ }_{i}$. This was already observed in Greco et al. (2001b, Theorem 2.3, page 125)

\subsection{Interpretations of model (D1)}

The framework offered by model $(D 1)$ is quite flexible. It contains many other sorting models as particular cases and may be reformulated in several interesting ways.

Consider first a conjunctive sorting model. In such a model an object belongs to $\mathscr{A}$ if all its evaluations are judged satisfactory. Formally, this leads to define, for all $i \in N$, a subset $\mathscr{A}_{i} \subseteq X_{i}$ containing the "satisfactory" levels on attribute $i$ and to declare that $x \in \mathscr{A}$ iff $x_{i} \in \mathscr{A}_{i}$, for all $i \in N$. Goldstein (1991, p. 65) noted that the conjunctive model is a particular 
case of model $(D 1)$. To prove this assertion, it suffices to define $u_{i}$ letting $u_{i}\left(x_{i}\right)=1$ if $x_{i} \in \mathscr{A}_{i}$ and $u_{i}\left(x_{i}\right)=0$ otherwise, and to take $F$ such that:

$$
F\left(u_{1}\left(x_{1}\right), u_{2}\left(x_{2}\right), \ldots, u_{n}\left(x_{n}\right)\right)=\sum_{i \in N} u_{i}\left(x_{i}\right)-(n-0.5) .
$$

A similar remark applies to the disjunctive sorting model in which, $x \in \mathscr{A}$ iff $x_{i} \in \mathscr{A}_{i}$, for some $i \in N$, defining the functions $u_{i}$ as above and taking:

$$
F\left(u_{1}\left(x_{1}\right), u_{2}\left(x_{2}\right), \ldots, u_{n}\left(x_{n}\right)\right)=\sum_{i \in N} u_{i}\left(x_{i}\right)-0.5 .
$$

A common model in the psychological literature on categorization is the independent clue model in which:

$$
x \in \mathscr{A} \Leftrightarrow \sum_{i \in N} \phi_{i}\left(x_{i}\right)>K,
$$

where $\phi_{i}$ are real valued functions on $X_{i}$ and $K$ is a threshold. As observed by Goldstein (1991, p. 66-67), such a model is a particular case of model $(A d d)$ and, hence, of model $(D 1)$. Indeed, it suffices to take $u_{i}=\phi_{i}-K / n$ to obtain model $(A d d)$.

Independent clue models, in turn, include models in which the objects in $\mathscr{A}$ are the objects in $X$ that are "close" to a prototype object, where distance is computed using a real-valued function $f_{i}$ on each attribute. Indeed, suppose that $P^{\mathscr{A}}=\left(P_{1}^{\mathscr{A}}, P_{2}^{\mathscr{A}}, \ldots, P_{n}^{\mathscr{A}}\right)$ is the prototype object of category $\mathscr{A}$. Letting $\phi_{i}\left(x_{i}\right)=-\left[f_{i}\left(x_{i}\right)-f_{i}\left(P_{i}^{\mathscr{A}}\right)\right]^{2}$, the independent clue model assign to $\mathscr{A}$ the objects having a squared distance to $P^{\mathscr{A}}$ less than $K$.

A related model postulates the existence of a prototype for each category and assign objects to a category if they are closer to the prototype object of this category than to the other prototype. Let $P^{\mathscr{A}}$ and $P^{\mathscr{U}}$ be the prototype objects of categories $\mathscr{A}$ and $\mathscr{U}$. Letting $\phi_{i}\left(x_{i}\right)=\left[f_{i}\left(x_{i}\right)-f_{i}\left(P_{i}^{\mathscr{A}}\right)\right]^{2}-\left[f_{i}\left(x_{i}\right)-\right.$ $\left.f_{i}\left(P_{i}^{\mathscr{U}}\right)\right]^{2}$, the independent clue model with $K=0$ assigns to $\mathscr{A}$ the objects that are closer to $P^{\mathscr{A}}$ than to $P^{\mathscr{U}}$. Such an interpretation of model $(D 1)$ might be useful to analyze sorting models explicitly using prototypes such as the ones proposed in Henriet (2000) and Perny (1998).

Greco et al. (2001b, Theorem 2.1, parts 3 and 4$)^{1}$ have proposed two equivalent reformulations of model $(D 1)$.

The first model suggested by Greco et al. (2001b, Theorem 2.1, Part 4) uses "at least" decision rules ${ }^{2}$. In this model, a complete and transitive relation $\mathcal{S}_{i}$ is supposed to be defined on each $X_{i}$. A decision rule $d$ consists

\footnotetext{
${ }^{1}$ Closely related results appear, without proof, in Słowiński et al. (2002, Theorem 2.1).

${ }^{2}$ It is also be possible to use, equivalently, what Greco et al. (2001b) call "at most" decision rules.
} 
in a subset $N^{d} \subseteq N$ of attributes and, for each $i \in N^{d}$, in an element $\delta_{i}^{d} \in X_{i}$. The syntax of the "at least" decision rule $d$ is the following:

$$
\left[x_{i} \mathcal{S}_{i} \delta_{i}^{d}, \forall i \in N^{d}\right] \Rightarrow x \in \mathscr{A} .
$$

A set of decision rules $\mathcal{D}$ is said to represent $\langle\mathscr{A}, \mathscr{U}\rangle$ if,

- for each $x \in \mathscr{A}$, there is at least one decision rule in $d \in \mathcal{D}$ that matches $x$, i.e., such that $x$ satisfies the premises of $d:\left[x_{i} \mathcal{S}_{i} \delta_{i}^{d}, \forall i \in N^{d}\right]$,

- for each $y \in \mathscr{U}$, there is no decision rule in $\mathcal{D}$ that matches $y$.

Greco et al. (2001b) have argued that a model based on decision rules may be preferable to a model based on a functional representation, in terms of simplicity and transparency (this fact is at the heart of the "rough set approach" to MCDM problems as presented in Greco et al. 1999, 2002b, 2005).

Greco et al. (2001b, Theorem 2.1, Part 4) show that the "at least" decision rule model holds iff $\langle\mathscr{A}, \mathscr{U}\rangle$ is linear.

\section{Remark 9}

Because the proof of the above fact is simple and instructive and it may not be easily accessible, we recall its main steps below.

It is simple to show that this model implies linearity. Indeed, suppose that $\left(x_{i}, a_{-i}\right) \in \mathscr{A}$ and $\left(y_{i}, b_{-i}\right) \in \mathscr{A}$, so that $\left(x_{i}, a_{-i}\right)$ is matched by rule $d^{1} \in \mathcal{D}$ and $\left(y_{i}, b_{-i}\right)$ is matched by rule $d^{2} \in \mathcal{D}$ (possibly, with $d^{1}=d^{2}$ ). If $i \notin N^{d^{1}}$ or if $i \notin N^{d^{2}}$, linearity cannot be violated. Suppose therefore that $i \in N^{d^{1}}$ and $i \in N^{d^{2}}$. Since the relations $\mathcal{S}_{i}$ are complete, we have either $x_{i} \mathcal{S}_{i} y_{i}$ or $y_{i} \mathcal{S}_{i} x_{i}$. Because $\left(x_{i}, a_{-i}\right)$ is matched by rule $d^{1} \in \mathcal{D}$ and $\left(y_{i}, b_{-i}\right)$ is matched by rule $d^{2} \in \mathcal{D}$, we know that $x_{i} \mathcal{S}_{i} \delta_{i}^{d^{1}}$ and $y_{i} \mathcal{S}_{i} \delta_{i}^{d^{2}}$. Because $\mathcal{S}_{i}$ are transitive, we have either that $y_{i} \mathcal{S}_{i} \delta_{i}^{d^{1}}$ or $x_{i} \mathcal{S}_{i} \delta_{i}^{d^{2}}$. Hence, either $\left(y_{i}, a_{-i}\right)$ is matched by rule $d^{1} \in \mathcal{D}$ or $\left(x_{i}, b_{-i}\right)$ is matched by rule $d^{2} \in \mathcal{D}$. This implies either $\left(y_{i}, a_{-i}\right) \in \mathscr{A}$ or $\left(x_{i}, b_{-i}\right) \in \mathscr{A}$, so that linear $r_{i}$ holds.

Conversely, suppose that $\langle\mathscr{A}, \mathscr{U}\rangle$ is linear. Using Lemma 5, we know that $\succsim_{i}$ is complete and we take, for all $i \in N, \mathcal{S}_{i}=\succsim_{i}$. For each $x \in \mathscr{A}$ define an "at least" decision rule $d^{x}$ saying that:

$$
\left[y_{i} \mathcal{S}_{i} x_{i}, \forall i \in N\right] \Rightarrow y \in \mathscr{A} \text {. }
$$

It is clear that each $x \in \mathscr{A}$ is matched by decision rule $d^{x}$. Suppose now that $y \in \mathscr{U}$ is matched by a decision rule. This would imply that, for some $x \in \mathscr{A}, y_{i} \succsim_{i} x_{i}$, for all $i \in N$. This implies $y \in \mathscr{A}$, a contradiction.

We refer the reader to Greco et al. (2001b); Słowiński et al. (2002) for an in depth study of the decision rule model for sorting and several of its extensions. 
Let us observe that it is not difficult to reformulate model $(D 0)$ in terms of decision rules. This time, the decision rules may have a syntax that is distinct from "at least" decision rules, since such a model does not imply linearity (for a general introduction to such models, see Pawlak, 1991).

The second model (henceforth "relational") proposed in Greco et al. (2001b, Theorem 2.1, Part 3) is based on binary relations. A complete and transitive relation $\mathcal{S}_{i}$ is supposed to be defined on each $X_{i}$. A reflexive binary relation $\mathcal{S}$ is defined on $X$ in such a way that it is compatible with the relations $\mathcal{S}_{i}$, i.e., such that, for all $x, y \in X$, all $i, j \in N$, all $z_{i} \in X_{i}$ and all $w_{j} \in X_{j}$,

$$
\left[x \mathcal{S} y, z_{i} \mathcal{S}_{i} x_{i}, y_{j} \mathcal{S}_{j} w_{j}\right] \Rightarrow\left(z_{i}, x_{-i}\right) \mathcal{S}\left(w_{j}, y_{-j}\right) .
$$

This expresses the fact that $\mathcal{S}$ is compatible with the dominance relation derived from the relations $\mathcal{S}_{i}$ (for a general study of such relations, see Bouyssou and Pirlot, 2004b). The relation $\mathcal{S}$ on $X$ is used to assign alternatives to $\mathscr{A}$ through their comparison with a particular element of $X$. More precisely, the relational model is such that:

$$
x \in \mathscr{A} \Leftrightarrow x \mathcal{S} \pi
$$

where $\pi \in X$ is interpreted as the lower limiting profile of category $\mathscr{A}$.

Greco et al. (2001b, Theorem 2.1, Part 3) have shown that the relational model holds iff $\langle\mathscr{A}, \mathscr{U}\rangle$ is linear.

\section{Remark 10}

Because the original text may not be easily accessible, we present below a simple proof of the above fact.

The necessity of linearity is easily shown. Indeed suppose that $\left(x_{i}, a_{-i}\right) \in$ $\mathscr{A}$ and $\left(y_{i}, b_{-i}\right) \in \mathscr{A}$, so that $\left(x_{i}, a_{-i}\right) \mathcal{S} \pi$ and $\left(y_{i}, b_{-i}\right) \mathcal{S} \pi$. Since the relations $\mathcal{S}_{i}$ are complete, we have either $x_{i} \mathcal{S}_{i} y_{i}$ or $y_{i} \mathcal{S}_{i} x_{i}$. Using (8), this implies that either $\left(y_{i}, a_{-i}\right) \mathcal{S} \pi$ or $\left(x_{i}, b_{-i}\right) \mathcal{S} \pi$. Hence, we have either $\left(y_{i}, a_{-i}\right) \in \mathscr{A}$ or $\left(x_{i}, b_{-i}\right) \in \mathscr{A}$, so that linear ${ }_{i}$ holds.

Conversely ${ }^{3}$ suppose that $\langle\mathscr{A}, \mathscr{U}\rangle$ is linear. We know that the relations $\succsim_{i}$ are weak orders and we take, for all $i \in N, \mathcal{S}_{i}=\succsim_{i}$.

Define a binary relation $R$ on $X$ letting, for all $x, y \in X, x R y$ iff $[x \in \mathscr{A}$ or $[x \in \mathscr{U}$ and $y \in \mathscr{U}]]$. It is easy to see that $R$ is a weak order on $X$ having two equivalence classes. Take $\mathcal{S}=R$. Define $\pi \in X$ to be any element of $\mathscr{A}$. We clearly have $x \in \mathscr{A} \Leftrightarrow x \mathcal{S} \pi$. It is easy to see that with such definitions (8) holds.

\footnotetext{
${ }^{3}$ Our proof differs from the one proposed in Greco et al. (2001b). When $X$ is not finite, the proof proposed by Greco et al. (2001b) would need to be adapted.
} 


\section{ELECTRE TRI}

Although many sorting methods using the concept of "outranking relation" have been proposed in the literature (see, e.g., Doumpos and Zopounidis, 2002, 2004; Figueira et al., 2004; Henriet, 2000; Massaglia and Ostanello, 1991; Moscarola and Roy, 1977; Norese and Viale, 2002; Perny, 1998; Roy, 1981), ELECTRE TRI appears to be the technique that has generated most studies (see Dias and Clímaco, 2000; Dias and Mousseau, 2006; Dias et al., 2002; Lourenco and Costa, 2004; Mousseau et al., 2001a; Mousseau and Słowiński, 1998; Ngo The and Mousseau, 2002; Tervonen et al., 2005) and applications (see, e.g., Andenmatten, 1995; Arondel and Girardin, 2000; Georgopoulou et al., 2003; Moussa, 2001; Mousseau et al., 2000a, 2001b; Roy, 2002). This motivates our study aiming at giving this method sound theoretical foundations.

For the ease of future reference, we briefly recall here the main points of the ELECTRE TRI sorting technique with two categories. For a more detailed description, we refer the reader to Mousseau et al. (2000b), Roy and Bouyssou (1993, ch. 6) or Wei (1992). We suppose below that preference and indifference thresholds are equal and that discordance effects occur in an "all or nothing" way. This will allow to keep things simple while preserving what we believe to be the general spirit of the method (note that under these hypotheses, there is no difference between the original version of ELECTRE TRI and the variant suggested in Mousseau and Dias (2004); furthermore, these hypotheses are quite reasonable when ELECTRE TRI is applied on a family of criteria that are all expressed on discrete scales).

Suppose that ELECTRE TRI is used to sort alternatives evaluated on several attributes into two ordered categories $\mathscr{A}$ and $\mathscr{U}$, with $\mathscr{A}$ containing the most desirable alternatives. This is done as follows. There is a profile $p$ being the lower limit of category $\mathscr{A}$ and the upper limit of $\mathscr{U}$. This profile $p$ is defined by its evaluations $\left(p_{1}, p_{2}, \ldots, p_{n}\right)$ on the attributes in $N$. Define $\widehat{X}_{i}=X_{i} \cup\left\{p_{i}\right\}$ and $\widehat{X}=\prod_{i=1}^{n} \widehat{X}_{i}$.

\section{Remark 11}

Observe that we do not suppose here that $p \in X$, in order to keep in line with the original description of the method. We shall later see (see Remarks 31 and 37 ) that it is not restrictive to consider a profile belonging to $X$.

For each $i \in N$, there is a semiorder $S_{i}$ on $\widehat{X}_{i}$. This relation is interpreted as an "at least as good" relation on $\widehat{X}_{i}$. A strict semiorder (i.e., an irreflexive, Ferrers and semi-transitive relation) $V_{i}$ is also defined on $\widehat{X}_{i}$. It is interpreted as a "far better than" relation on $\widehat{X}_{i}$. For consistency reasons, it is supposed that $V_{i}$ is included in the asymmetric part of $S_{i}$. 
A nonnegative weight $w_{i}$ is assigned to each attribute $i \in N$. We suppose w.l.o.g. that weights are normalized so that $\sum_{i=1}^{n} w_{i}=1$. Let $\lambda$ be a real number between $1 / 2$ and 1 .

In ELECTRE TRI, a binary relation $S$ is built on $\widehat{X}$ letting, for all $x, y \in \widehat{X}$ (notice that it would be enough to define $S$ as a relation between the sets $X$ and $\{p\})$,

$$
x S y \Leftrightarrow \sum_{i \in S(x, y)} w_{i} \geq \lambda \text { and }\left[\operatorname{Not}\left[y_{i} V_{i} x_{i}\right], \text { for all } i \in N\right],
$$

where $S(x, y)=\left\{i \in N: x_{i} S_{i} y_{i}\right\}$. Hence, we have $x S y$ when $x$ is judged "at least as good as" $y$ on a qualified weighted majority of attributes (concordance condition) and there is no attribute on which $y$ is judged "far better" than $x$ (non-discordance condition).

The sorting of an alternative $x \in X$ is based upon the comparison of $x$ with the profile $p$ using the relation $S$. In the pessimistic version of ELECTRE TRI, we have, for all $x \in X$,

$$
x \in \mathscr{A} \Leftrightarrow x S p
$$

In the optimistic version of ELECTRE TRI, we have, for all $x \in X$,

$$
x \in \mathscr{A} \Leftrightarrow \operatorname{Not}[p P x],
$$

where $P$ is the asymmetric part of $S$.

Let us note that if we have $x \in \mathscr{A}$ in the pessimistic version of ELECTRE TRI, we have $x S p$ so that $\operatorname{Not}\left[\begin{array}{lll}p & P & x\end{array}\right]$. Hence, we must have $x \in \mathscr{A}$ with the optimistic version of ELECTRE TRI. This explains the names of the two versions of the method.

It is not difficult to show that a partition $\langle\mathscr{A}, \mathscr{U}\rangle$ has been obtained using the pessimistic version of ELECTRE TRI must be linear. Our central aim will be to investigate what must be added to linearity in order to obtain a model that is as close as possible to ELECTRE TRI.

\section{The noncompensatory sorting model for twofold partitions}

\subsection{Definitions}

We say that $\langle\mathscr{A}, \mathscr{U}\rangle$ has a representation in the noncompensatory sorting model if: 
- for all $i \in N$ there is a set $\mathscr{A}_{i} \subseteq X_{i}$,

- there is a subset $\mathscr{F}$ of $2^{N}$ such that, for all $I, J \in 2^{N}$,

$$
[I \in \mathscr{F} \text { and } I \subseteq J] \Rightarrow J \in \mathscr{F},
$$

such that, for all $x \in X$,

$$
x \in \mathscr{A} \Leftrightarrow\left\{i \in N: x_{i} \in \mathscr{A}_{i}\right\} \in \mathscr{F} .
$$

In this case, we say, that $\left\langle\mathscr{F}, \mathscr{A}_{1}, \mathscr{A}_{2}, \ldots, \mathscr{A}_{n}\right\rangle$ or, for short, $\left\langle\mathscr{F},\left\langle\mathscr{A}_{i}\right\rangle_{i \in N}\right\rangle$ is a representation of $\langle\mathscr{A}, \mathscr{U}\rangle$ in the noncompensatory sorting model. When there is no risk of confusion on the underlying sets $\mathscr{A}_{i}$, we write $A(x)$ instead of $\left\{i \in N: x_{i} \in \mathscr{A}_{i}\right\}$. In this section, we write $\mathscr{U}_{i}=X_{i} \backslash \mathscr{A}_{i}$.

We may interpret the noncompensatory sorting model as follows. On each $i \in N$, we isolate, within the set $X_{i}$ a set $\mathscr{A}_{i}$ of "satisfactory" levels. In order for an alternative $x \in X$ to be globally satisfactory, i.e., that $x \in \mathscr{A}$, it is necessary and sufficient that the evaluations of $x$ are judged satisfactory on a subset of attributes that is "sufficiently important", as indicated by the set $\mathscr{F}$. Condition (11) on $\mathscr{F}$ means that replacing an evaluation in $\mathscr{U}_{i}=$ $X_{i} \backslash \mathscr{A}_{i}$ by an evaluation in $\mathscr{A}_{i}$ cannot turn a satisfactory alternative into an unsatisfactory one. It is obvious that this model generalizes the conjunctive and disjunctive models introduced above by admitting more complex sets $\mathscr{F}$.

\section{Remark 12}

The interpretation of the noncompensatory sorting model suggested above should not hide the fact that the roles of $\mathscr{A}$ and $\mathscr{U}$ are entirely symmetric in the noncompensatory sorting model. Indeed, suppose that $\langle\mathscr{A}, \mathscr{U}\rangle$ has a representation $\left\langle\mathscr{F},\left\langle\mathscr{A}_{i}\right\rangle_{i \in N}\right\rangle$ in the noncompensatory sorting model. Let $U(x)=\left\{i \in N: x_{i} \in \mathscr{U}_{i}\right\}$ and define $\mathscr{G} \subseteq 2^{N}$ letting $I \in \mathscr{G} \Leftrightarrow N \backslash I \notin \mathscr{F}$. It is clear that $\mathscr{G}$ satisfies (11) and that

$$
x \in \mathscr{U} \Leftrightarrow U(x) \in \mathscr{G} .
$$

Hence, interpreting the set $\mathscr{A}$ as containing satisfactory alternatives is arbitrary.

The rationale for the name "noncompensatory" comes from the fact that these sorting models do not distinguish more than two types of elements in $X_{i}$ : those in $\mathscr{A}_{i}$ and those in $\mathscr{U}_{i}$. Suppose that $x$ is not in $\mathscr{A}$ because $A(x)$ does not belong to $\mathscr{F}$. In a compensatory model, it would be possible to improve the assignment of $x$ by sufficiently improving its evaluation on any attribute. In the noncompensatory sorting model, altering the evaluation of 
$x$ on any attribute in $A(x)$ will never lead to modify the assignment of $x$ in $\mathscr{U}$.

As pointed out to us by Salvatore Greco, Benedetto Matarazzo and Roman Słowiński, the noncompensatory sorting model has close connections with the Sugeno integral model for sorting that has been analyzed in Greco et al. (2001b) and Słowiński et al. (2002). They will be detailed in section 5.4 below. Suppose that $\langle\mathscr{A}, \mathscr{U}\rangle$ has a representation $\left\langle\mathscr{F},\left\langle\mathscr{A}_{i}\right\rangle_{i \in N}\right\rangle$ in the noncompensatory sorting model and that $i \in N$ is influent so that $\left(x_{i}, a_{-i}\right) \in \mathscr{A}$ and $\left(y_{i}, a_{-i}\right) \notin \mathscr{A}$, for some $x_{i}, y_{i} \in X_{i}$ and some $a_{-i} \in X_{-i}$. Using the definition of the noncompensatory sorting model, we must have $x_{i} \in \mathscr{A}_{i}$ and $y_{i} \in \mathscr{U}_{i}$, so that $\varnothing \subsetneq \mathscr{A}_{i} \subsetneq X_{i}$. When $\langle\mathscr{A}, \mathscr{U}\rangle$ has a representation in the noncompensatory sorting model and all attributes are influent for $\langle\mathscr{A}, \mathscr{U}\rangle$, this implies that, for all $I \subseteq N$, we have $A(x)=I$, for some $x \in X$.

It is not difficult to see that the representation of $\langle\mathscr{A}, \mathscr{U}\rangle$ in the noncompensatory sorting model is unique as soon as each attribute is influent for $\langle\mathscr{A}, \mathscr{U}\rangle$. Indeed, suppose that $\left\langle\mathscr{F},\left\langle\mathscr{A}_{i}\right\rangle_{i \in N}\right\rangle$ and $\left\langle\mathscr{F}^{\prime},\left\langle\mathscr{A}_{i}^{\prime}\right\rangle_{i \in N}\right\rangle$ are two distinct representations of $\langle\mathscr{A}, \mathscr{U}\rangle$. Suppose that $x_{i} \in \mathscr{A}_{i}$ and $x_{i} \notin \mathscr{A}_{i}^{\prime}$. Because $i \in N$ is influent we know that there are $z_{i}, w_{i} \in X_{i}$ and $a_{-i} \in X_{-i}$ such that $\left(w_{i}, a_{-i}\right) \in \mathscr{U}$ and $\left(z_{i}, a_{-i}\right) \in \mathscr{A}$. Since $\left(z_{i}, a_{-i}\right) \in \mathscr{A}$ and $x_{i} \in \mathscr{A}_{i}$, we must have $\left(x_{i}, a_{-i}\right) \in \mathscr{A}$. Similarly, $\left(w_{i}, a_{-i}\right) \in \mathscr{U}$ and $x_{i} \in \mathscr{U}_{i}^{\prime}$ imply $\left(x_{i}, a_{-i}\right) \in \mathscr{U}$, a contradiction. We must therefore have $\mathscr{A}_{i}=\mathscr{A}_{i}^{\prime}$, for all $i \in N$. This implies $\mathscr{F}=\mathscr{F}^{\prime}$ so that the representation of $\langle\mathscr{A}, \mathscr{U}\rangle$ is unique.

The following example illustrates the construction of a representation of $\langle\mathscr{A}, \mathscr{U}\rangle$ in the noncompensatory sorting model.

\section{Example 13}

Suppose that $n=3, X_{1}=X_{2}=X_{3}=\{9,10,11\}$. Let $\mathscr{A}=\{(10,9,10)$, $(10,9,11),(10,10,9),(10,10,10),(10,10,11),(10,11,9),(10,11,10),(10,11,11)$, $(11,9,10),(11,9,11),(11,10,9),(11,10,10),(11,10,11),(11,11,9),(11,11,10)$, $(11,11,11)\}$ and $\mathscr{U}=\{(9,9,9),(9,9,10),(9,9,11),(9,10,9),(9,10,10)$, $(9,10,11),(9,11,9),(9,11,10),(9,11,11),(10,9,9),(11,9,9)\}$.

Attribute 1 is influent because, e.g., $(10,9,10) \in \mathscr{A}$ but $(9,9,10) \in \mathscr{U}$. Attribute 2 is influent because, e.g., $(10,10,9) \in \mathscr{A}$ but $(10,9,9) \in \mathscr{U}$. Similarly, attribute 3 is influent because, e.g., $(10,9,10) \in \mathscr{A}$ but $(10,9,9) \in$ $\mathscr{U}$.

It is not difficult to check that $\langle\mathscr{A}, \mathscr{U}\rangle$ has a unique representation with $\mathscr{A}_{1}=\mathscr{A}_{2}=\mathscr{A}_{3}=\{10,11\}$ and $\mathscr{F}=\{\{1,2\},\{1,3\},\{1,2,3\}\}$. This is detailed in Table 1. 


\begin{tabular}{cccccc}
$x$ & Cat. & $A(x)$ & $x$ & Cat. & $A(x)$ \\
\hline$(10,9,10)$ & $\mathscr{A}$ & $\{1,3\}$ & $(9,9,9)$ & $\mathscr{U}$ & $\varnothing$ \\
$(10,9,11)$ & $\mathscr{A}$ & $\{1,3\}$ & $(9,9,10)$ & $\mathscr{U}$ & $\{3\}$ \\
$(10,10,9)$ & $\mathscr{A}$ & $\{1,2\}$ & $(9,9,11)$ & $\mathscr{U}$ & $\{3\}$ \\
$(10,10,10)$ & $\mathscr{A}$ & $\{1,2,3\}$ & $(9,10,9)$ & $\mathscr{U}$ & $\{2\}$ \\
$(10,10,11)$ & $\mathscr{A}$ & $\{1,2,3\}$ & $(9,10,10)$ & $\mathscr{U}$ & $\{2,3\}$ \\
$(10,11,9)$ & $\mathscr{A}$ & $\{1,2\}$ & $(9,10,11)$ & $\mathscr{U}$ & $\{2,3\}$ \\
$(10,11,10)$ & $\mathscr{A}$ & $\{1,2,3\}$ & $(9,11,9)$ & $\mathscr{U}$ & $\{2\}$ \\
$(10,11,11)$ & $\mathscr{A}$ & $\{1,2,3\}$ & $(9,11,10)$ & $\mathscr{U}$ & $\{2,3\}$ \\
$(11,9,10)$ & $\mathscr{A}$ & $\{1,3\}$ & $(9,11,11)$ & $\mathscr{U}$ & $\{2,3\}$ \\
$(11,9,11)$ & $\mathscr{A}$ & $\{1,3\}$ & $(10,9,9)$ & $\mathscr{U}$ & $\{1\}$ \\
$(11,10,9)$ & $\mathscr{A}$ & $\{1,2\}$ & $(11,9,9)$ & $\mathscr{U}$ & $\{1\}$ \\
$(11,10,10)$ & $\mathscr{A}$ & $\{1,2,3\}$ & & & \\
$(11,10,11)$ & $\mathscr{A}$ & $\{1,2,3\}$ & & & \\
$(11,11,9)$ & $\mathscr{A}$ & $\{1,2\}$ & & & \\
$(11,11,10)$ & $\mathscr{A}$ & $\{1,2,3\}$ & & & \\
$(11,11,11)$ & $\mathscr{A}$ & $\{1,2,3\}$ & & &
\end{tabular}

Table 1: Details of Example 13

All attributes are influent, $\langle\mathscr{A}, \mathscr{U}\rangle$ has a unique representation in which $\mathscr{A}_{1}=\mathscr{A}_{2}=\mathscr{A}_{3}=\{10,11\}$ and $\mathscr{F}=\{\{1,2\},\{1,3\},\{1,2,3\}\}$.

\subsection{Particular cases}

The noncompensatory sorting model is sufficiently flexible to include several sorting models of particular interest. Suppose that $\langle\mathscr{A}, \mathscr{U}\rangle$ has a representation $\left\langle\mathscr{F},\left\langle\mathscr{A}_{i}\right\rangle_{i \in N}\right\rangle$ in the noncompensatory sorting model. If $\mathscr{F}=\{N\}$, we clearly obtain the conjunctive sorting model. Similarly, if, for all $i \in N$, $\{i\} \in \mathscr{F}$, so that $\mathscr{F}=2^{N} \backslash\{\varnothing\}$, we obtain the disjunctive sorting model. Suppose that a positive weight $w_{i}$ can be attached to each attribute $i \in N$ in such a way that:

$$
I \in \mathscr{F} \Leftrightarrow \sum_{i \in I} w_{i} \geq \lambda
$$

where $\lambda$ is a positive threshold. This gives a a model in which an alternative is satisfactory when it is satisfactory on a qualified weighted majority of attributes.

The above three particular cases of the noncompensatory sorting model are quite familiar. They were thoroughly discussed in Fishburn (1978).

The pessimistic version of ELECTRE TRI, when preference and indifference thresholds are equal and when discordance is not involved (i.e., $V_{i}=\varnothing$, for all $i \in N$ ), is a particular case of the noncompensatory sorting model. 
Using the notation of section 4 , we have, for all $x \in X$,

$$
x \in \mathscr{A} \Leftrightarrow x S p \Leftrightarrow \sum_{i \in S(x, p)} w_{i} \geq \lambda .
$$

Defining $\mathscr{A}_{i}=\left\{x_{i} \in X_{i}: x_{i} S_{i} p_{i}\right\}$ and letting $I \in \mathscr{F}$ when $\sum_{i \in I} w_{i} \geq \lambda$ shows that such a model is a particular case of the noncompensatory sorting model. For instance, the reader might want to check that the partition $\langle\mathscr{A}, \mathscr{U}\rangle$ used in Example 13 above can be obtained using the pessimistic version of ELECTRE TRI with $p=(10,10,10), V_{i}=\varnothing$ and $S_{i}=\geq$ for all $i \in N, w_{1}=0.5, w_{2}=w_{3}=0.25$ and $\lambda=2 / 3$.

In the optimistic version of ELECTRE TRI, when preference and indifference thresholds are equal and when discordance is not involved, we have, for all $x \in X$,

$$
x \in \mathscr{A} \Leftrightarrow \operatorname{Not}\left[\begin{array}{lll}
p & P & x
\end{array}\right] \Leftrightarrow\left[\sum_{i \in S(p, x)} w_{i}<\lambda \text { or } \sum_{i \in S(x, p)} w_{i} \geq \lambda\right] .
$$

Beyond surface, the two versions of the ELECTRE TRI method are rather different. Indeed, as shown by the following example, due to its use of $P$, the optimistic version of ELECTRE TRI does not fit into the framework of the noncompensatory sorting model.

\section{Example 14}

Suppose that $n=5, X_{1}=X_{2}=\ldots=X_{5}=\{9,10,11\}$ and $p=(10,10$, $10,10,10)$. For all $i \in\{1,2, \ldots, 5\}$, let $w_{i}=1 / 5, V_{i}=\varnothing$ and $S_{i}=\geq$. Let $\lambda=4 / 5$.

Using the optimistic version of ELECTRE TRI, we obtain $x=(11,11,9$, $9,9) \in \mathscr{A}$ (we have $\operatorname{Not}[p S x]$ and $\operatorname{Not}[x S p]$ ), $y=(10,11,9,9,9) \in \mathscr{U}$ (we have $p S y$ and $\operatorname{Not}[y S p]$ ) and $z=(10,10,10,10,10) \in \mathscr{A}$ (we have $p S z$ and $z S p$ ).

Suppose that this partition can be represented in the noncompensatory sorting model. Since $x \in \mathscr{A}$ and $y \in \mathscr{U}$, we must have $10 \in \mathscr{U}_{1}$ and $11 \in \mathscr{A}_{1}$. The problem being entirely symmetric, we easily obtain that, for all $i \in N$, $10 \in \mathscr{U}_{i}$ and $11 \in \mathscr{A}_{i}$. Because $z \in \mathscr{A}$, we must have $\varnothing \in \mathscr{F}$, so that all alternatives in $X$ should belong to $\mathscr{A}$. This is clearly false. Hence, this partition cannot be represented in the noncompensatory sorting model.

\subsection{Axioms and results}

Let us first observe that if $\langle\mathscr{A}, \mathscr{U}\rangle$ has a representation in the noncompensatory sorting model then it must be linear and, for all $i \in N$, the weak order $\succsim_{i}$ can have at most two distinct equivalence classes. 


\section{Lemma 15}

Suppose that $\langle\mathscr{A}, \mathscr{U}\rangle$ has a representation in the noncompensatory sorting model. Then, for all $i \in N$,

1. condition linear ${ }_{i}$ holds, so that $\succsim_{i}$ is a weak order,

2. the weak order $\succsim_{i}$ can have at most two distinct equivalence classes.

PROOF

Part 1. Suppose that $\left(x_{i}, a_{-i}\right) \in \mathscr{A}$ and $\left(y_{i}, b_{-i}\right) \in \mathscr{A}$. If $x_{i} \in \mathscr{A}_{i}$, we have $A\left(\left(y_{i}, b_{-i}\right)\right) \subseteq A\left(\left(x_{i}, b_{-i}\right)\right)$ so that $\left(x_{i}, b_{-i}\right) \in \mathscr{A}$, using condition (11). If $x_{i} \in \mathscr{U}_{i}$, we have $A\left(\left(x_{i}, a_{-i}\right)\right) \subseteq A\left(\left(y_{i}, a_{-i}\right)\right)$ so that $\left(y_{i}, a_{-i}\right) \in \mathscr{A}$.

Part 2. Suppose that $\left(x_{i}, a_{-i}\right) \in \mathscr{A}$ and $\operatorname{Not}\left[\left(y_{i}, a_{-i}\right) \in \mathscr{A}\right]$, so that $x_{i}$ and $y_{i}$ must belong to distinct equivalence classes of $\succsim_{i}$. This implies $x_{i} \in \mathscr{A}_{i}$ and $y_{i} \in \mathscr{U}_{i}$. Using the definition of the noncompensatory sorting model, any $z_{i} \in X_{i}$ must belong either to $\mathscr{A}_{i}$ or $\mathscr{U}_{i}$. This implies that we have either $x_{i} \sim_{i} z_{i}$ or $y_{i} \sim_{i} z_{i}$.

The following simple proposition shows how the noncompensatory sorting model can be represented in model $(D 1)$.

\section{Proposition 16}

$\langle\mathscr{A}, \mathscr{U}\rangle$ has a representation in the noncompensatory sorting model iff it has a representation in model (D1) in which each $u_{i}$ takes at most two distinct values.

\section{ProOF}

Since, in model $(D 1)$, it is always possible to take $u_{i}$ as a numerical representation of $\succsim_{i}$, necessity results from Lemma 15 and Proposition 6 . In order to show sufficiency, suppose that $\langle\mathscr{A}, \mathscr{U}\rangle$ has a representation in model $(D 1)$ in which each $u_{i}$ takes at most two distinct values. It can always be supposed, in view of the uniqueness of the representation in model $(D 1)$, that, when $u_{i}$ takes exactly two values, these are 0 and 1 and, when $u_{i}$ takes exactly one value, this value is 0 . For all $i \in N$, define $\mathscr{A}_{i}$ letting $x_{i} \in \mathscr{A}_{i}$ iff $u_{i}\left(x_{i}\right)=1$, so that $A(x)=\left\{i \in N: u_{i}\left(x_{i}\right)=1\right\}$. Define $\mathscr{F}$ letting, for all $I \in 2^{N}, I \in \mathscr{F}$ if $A(x) \subseteq I$, for some $x \in \mathscr{A}$. Because $\mathscr{A} \neq \varnothing$, there must be at least one attribute for which $u_{i}$ takes two distinct values. Hence, $\mathscr{F} \neq \varnothing$, so that it is satisfies (11). Clearly if $x \in \mathscr{A}$, we have $A(x) \in \mathscr{F}$. Conversely, suppose that $A(x) \in \mathscr{F}$, so that, for some $y \in \mathscr{A}, A(y) \subseteq A(x)$. Given the definition of the sets $\mathscr{A}_{i}$, this implies $x_{i} \succsim_{i} y_{i}$, for all $i \in N$, so that $x \in \mathscr{A}$.

In view of Proposition 15, a characterization of the noncompensatory sorting model will be at hand if we add to linearity a condition implying that all 
relations $\succsim_{i}$ have at most two equivalence classes. We say that $\langle\mathscr{A}, \mathscr{U}\rangle$ is 2 -graded on attribute $i \in N\left(\right.$ condition 2 -graded $\left._{i}\right)$ if

$$
\left.\begin{array}{l}
\left(x_{i}, a_{-i}\right) \in \mathscr{A} \\
\text { and } \\
\left(y_{i}, a_{-i}\right) \in \mathscr{A} \\
\text { and } \\
\left(y_{i}, b_{-i}\right) \in \mathscr{A}
\end{array}\right\} \Rightarrow\left\{\begin{array}{c}
\left(x_{i}, b_{-i}\right) \in \mathscr{A} \\
\text { or } \\
\left(z_{i}, a_{-i}\right) \in \mathscr{A}, \quad(2 \text { - }, \quad
\end{array}\right.
$$

for all $x_{i}, y_{i}, z_{i} \in X_{i}$ and all $a_{-i}, b_{-i} \in X_{-i}$. We say that $\langle\mathscr{A}, \mathscr{U}\rangle$ is 2-graded if it is 2-graded on all attributes $i \in N$. Condition 2-graded is inspired by related works on noncompensatory models in the context of binary relations by Bouyssou and Pirlot (2002a, 2005) and Greco et al. (2001a). The intuition behind this condition is simple. Suppose that $\left(y_{i}, b_{-i}\right) \in \mathscr{A}$ and $\operatorname{Not}\left[\left(x_{i}, b_{-i}\right) \in \mathscr{A}\right]$. If linear $i_{i}$ holds, this implies that $y_{i} \succ_{i} x_{i}$. If the relation $\succsim_{i}$ only has two distinct equivalence classes, this implies that, for all $z_{i} \in X_{i}$, we have $z_{i} \succsim_{i} x_{i}$. Therefore if $\left(x_{i}, a_{-i}\right) \in \mathscr{A}$, we must have $\left(z_{i}, a_{-i}\right) \in \mathscr{A}$, as required by condition 2 -graded $_{i}$. The role of the additional premise $\left(y_{i}, a_{-i}\right) \in \mathscr{A}$ is to ensure that condition 2 -graded $_{i}$ is independent

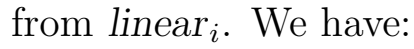

\section{Lemma 17}

1. Conditions linear $i$ and 2 -graded $_{i}$ hold iff $\succsim_{i}$ is a weak order having at most two distinct equivalence classes.

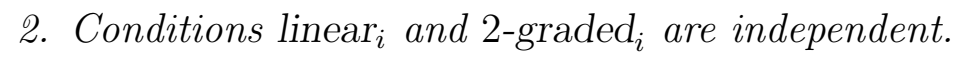

\section{ProOF}

Part 1. Necessity. The necessity of linear ${ }_{i}$ follows from Part 1 of lemma 15. Suppose that 2 graded $_{i}$ is violated so that, for some $x_{i}, y_{i}, z_{i} \in X_{i}$ and some $a_{-i}, b_{-i} \in X_{-i}$, we have $\left(x_{i}, a_{-i}\right) \in \mathscr{A},\left(y_{i}, a_{-i}\right) \in \mathscr{A},\left(y_{i}, b_{-i}\right) \in \mathscr{A},\left(x_{i}, b_{-i}\right) \in$ $\mathscr{U}$ and $\left(z_{i}, a_{-i}\right) \in \mathscr{U}$. Using the definition of $\succsim_{i}$ this implies $\operatorname{Not}\left[x_{i} \succsim_{i} y_{i}\right]$, $\operatorname{Not}\left[z_{i} \succsim_{i} x_{i}\right]$ and $\operatorname{Not}\left[z_{i} \succsim_{i} y_{i}\right]$ implying that $\succsim_{i}$ has at least three distinct equivalence classes.

Sufficiency. Using linear ${ }_{i}$, we know that $\succsim_{i}$ is a weak order. Suppose that $\succsim_{i}$ has at least three equivalence classes so that, for some $x_{i}, y_{i}, z_{i} \in X_{i}$, we have $x_{i} \succ_{i} y_{i}$ and $y_{i} \succ_{i} z_{i}$. Using the definition of $\succsim_{i}$, we have, for some $a_{-i}, b_{-i} \in X_{-i},\left(x_{i}, a_{-i}\right) \in \mathscr{A},\left(y_{i}, a_{-i}\right) \in \mathscr{U},\left(y_{i}, b_{-i}\right) \in \mathscr{A},\left(z_{i}, b_{-i}\right) \in \mathscr{U}$. Using linear ${ }_{i},\left(x_{i}, a_{-i}\right) \in \mathscr{A},\left(y_{i}, b_{-i}\right) \in \mathscr{A}$ and $\left(y_{i}, a_{-i}\right) \in \mathscr{U}$ imply $\left(x_{i}, b_{-i}\right) \in$ $\mathscr{A}$. Using 2-graded $i,\left(y_{i}, b_{-i}\right) \in \mathscr{A},\left(x_{i}, b_{-i}\right) \in \mathscr{A}$ and $\left(x_{i}, a_{-i}\right) \in \mathscr{A}$ imply, either $\left(y_{i}, a_{-i}\right) \in \mathscr{A}$ or $\left(z_{i}, b_{-i}\right) \in \mathscr{A}$, a contradiction.

Part 2. Taking an additive model for sorting, examples of partitions that are linear and satisfy 2 -graded $i$ on all but one attribute are easy to build. 
Let us give an example of a partition $\langle\mathscr{A}, \mathscr{U}\rangle$ that is 2-graded and satisfies linear $_{i}$ on all but one attribute.

\section{Example 18 (2-graded and Not[1inear $\left.{ }_{i}\right]$ )}

Let $n=3$ and $X=\left\{x_{1}, y_{1}\right\} \times\left\{x_{2}, y_{2}\right\} \times\left\{x_{3}, y_{3}\right\}$. Let $\mathscr{A}=\left\{\left(x_{1}, x_{2}, x_{3}\right)\right.$, $\left.\left(x_{1}, y_{2}, x_{3}\right),\left(y_{1}, y_{2}, x_{3}\right),\left(y_{1}, y_{2}, y_{3}\right)\right\}$. Condition linear ${ }_{1}$ is violated since $\left(x_{1}\right.$, $\left.x_{2}, x_{3}\right) \in \mathscr{A}$ and $\left(y_{1}, y_{2}, y_{3}\right) \in \mathscr{A}$ but $\left(y_{1}, x_{2}, x_{3}\right) \notin \mathscr{A}$ and $\left(x_{1}, y_{2}, y_{3}\right) \notin \mathscr{A}$. We have $y_{2} \succ_{2} x_{2}$ and $x_{3} \succ_{3} y_{3}$, so that conditions linear ${ }_{2}$ and linear 3 hold. In order to show that $\langle\mathscr{A}, \mathscr{U}\rangle$ is 2 -graded, it suffices to observe that condition 2 graded $_{i}$ is trivially satisfied when $X_{i}$ has only two elements.

\section{Remark 19}

The conjunction of conditions linear $_{i}$ and 2 -graded $_{i}$ can be captured by a single condition. This condition was proposed in Słowiński et al. (2002, Theorem 2.4) in the apparently different context of the study of partitions that can be represented using a Sugeno integral. We shall shortly see that these two analyses are intimately related. For the time being, we note the following:

\section{Lemma 20}

$\langle\mathscr{A}, \mathscr{U}\rangle$ satisfies conditions linear ${ }_{i}$ and 2 -graded $_{i}$ if and only if it satisfies

$$
\left.\begin{array}{c}
\left(x_{i}, a_{-i}\right) \in \mathscr{A} \\
\text { and } \\
\left(y_{i}, b_{-i}\right) \in \mathscr{A}
\end{array}\right\} \Rightarrow\left\{\begin{array}{c}
\left(x_{i}, b_{-i}\right) \in \mathscr{A} \\
\text { or } \\
\left(z_{i}, a_{-i}\right) \in \mathscr{A}
\end{array}\right.
$$

for all $x_{i}, y_{i}, z_{i} \in X_{i}$ and all $a_{-i}, b_{-i} \in X_{-i}$.

\section{PROOF}

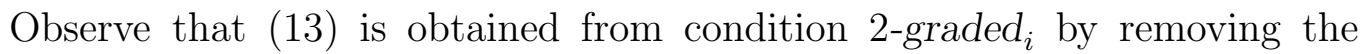
premise $\left(y_{i}, a_{-i}\right) \in \mathscr{A}$, so that it implies 2 -graded $_{i}$. Taking $z_{i}=y_{i}$ in the expression of (13) shows that it implies linear . $_{\text {. }}$

Conversely, suppose that $\left(x_{i}, a_{-i}\right) \in \mathscr{A}$ and $\left(y_{i}, b_{-i}\right) \in \mathscr{A}$. If $\left(y_{i}, a_{-i}\right) \in$ $\mathscr{A}, 2$ graded $_{i} \operatorname{implies}\left(x_{i}, b_{-i}\right) \in \mathscr{A}$ or $\left(z_{i}, a_{-i}\right) \in \mathscr{A}$. If $\left(y_{i}, a_{-i}\right) \notin \mathscr{A}$, $\left(x_{i}, a_{-i}\right) \in \mathscr{A}$ and $\left(y_{i}, b_{-i}\right) \in \mathscr{A}$ imply, using $\operatorname{linear}_{i},\left(x_{i}, b_{-i}\right) \in \mathscr{A}$.

Our main result in this section says that linearity and 2-gradedness characterize the noncompensatory sorting model for twofold partitions.

\section{Theorem 21}

A partition $\langle\mathscr{A}, \mathscr{U}\rangle$ has a representation in the noncompensatory sorting model iff it is linear and 2-graded. 


\section{PROOF}

Necessity results from lemmas 15 and 17.

Using lemma 17, we know that $\succsim_{i}$ is a weak order having at most two equivalence classes, so that $X_{i} / \sim_{i}$ is finite. Hence, taking an element from each of the equivalence class of $\sim_{i}$ gives a finite set $X_{i}^{\prime} \subseteq X_{i}$ that is dense in $X_{i}$ for $\succsim_{i}$. Using Proposition 6 , we know that $\langle\mathscr{A}, \mathscr{U}\rangle$ has a representation in model $(D 1)$ in which, for all $i \in N, u_{i}$ is a numerical representation of $\succsim_{i}$. Sufficiency therefore results from Proposition 16.

\section{Remark 22}

We have based the above sufficiency proof on Proposition 16 that uses a numerical representation in model $(D 1)$. A direct proof that does not appeal to the model $(D 1)$ is easy to devise.

Indeed, from Lemma 17 , we know that $\succsim_{i}$ is a weak order having at most two equivalence classes If, for some $i \in N, \succsim_{i}$ has exactly two distinct equivalence classes, define $\mathscr{A}_{i}$ as the elements of $X_{i}$ in the first equivalence class. If $\succsim_{i}$ has only one equivalence class, define $\mathscr{A}_{i}=\varnothing$. Define $\mathscr{F}$ letting $I \in \mathscr{F}$ whenever there is some $x \in \mathscr{A}$ such that $A(x) \subseteq I$. By hypothesis, $\mathscr{A} \neq \varnothing$ so that $\mathscr{F} \neq \varnothing$. Hence, $\mathscr{F}$ satisfies (11).

Let us show that $\left\langle\mathscr{F},\left\langle\mathscr{A}_{i}\right\rangle_{i \in N}\right\rangle$ is a representation of $\langle\mathscr{A}, \mathscr{U}\rangle$ in the noncompensatory sorting model. If $x \in \mathscr{A}$, we have $A(x) \in \mathscr{F}$. Conversely, suppose that $A(x) \in \mathscr{F}$. This implies that, for some $y \in \mathscr{A}$, we have $A(y) \subseteq A(x)$. Using the definition of the sets $\mathscr{A}_{i}, A(y) \subseteq A(x)$ implies $x_{i} \succsim_{i} y_{i}$, for all $i \in N$, so that $x \in \mathscr{A}$.

\subsection{The noncompensatory sorting model and the Sugeno integral}

Salvatore Greco, Benedetto Matarazzo and Roman Słowiński have brought to our attention the fact that Theorem 21 has very close connections with Theorem 2.4 stated, without proof, in Słowiński et al. (2002) (our results have been obtained independently). The purpose of Słowiński et al. (2002, Theorem 2.4) is to characterize partitions that can be represented using a Sugeno integral.

Let $\langle\mathscr{A}, \mathscr{U}\rangle$ be a partition of $X$. We say that $\langle\mathscr{A}, \mathscr{U}\rangle$ can be represented using a Sugeno integral, if there are:

- a non-negative real valued function $f_{i}$ on $X_{i}$, for all $i \in N$,

- a real valued function ${ }^{4} \mu$ on $2^{N}$ that is nondecreasing w.r.t. inclusion

\footnotetext{
${ }^{4}$ The function $\mu$ is usually called a capacity. It is often supposed that the capacity is normalized so that $\mu(N)=1$. This is not necessary for our purposes.
} 
(i.e., such that $A \subseteq B$ implies $\mu(A) \leq \mu(B))$ and such that $\mu(\varnothing)=0$, such that, for all $x \in X$,

$$
x \in \mathscr{A} \Leftrightarrow S_{\left\langle\mu, f_{i}\right\rangle}(x)=\bigvee_{I \subseteq N}\left[\bigwedge_{i \in I}\left[f_{i}\left(x_{i}\right)\right] ; \mu(I)\right]>0
$$

where $S_{\left\langle\mu, f_{i}\right\rangle}(x)$ is called the (discrete) Sugeno integral of the vector $\left(f_{1}\left(x_{1}\right)\right.$, $\left.f_{2}\left(x_{2}\right), \ldots, f_{n}\left(x_{n}\right)\right)$ w.r.t. the capacity $\mu$. We refer the reader to Marichal (2000) for a detailed study of various equivalent forms of $S_{\left\langle\mu, f_{i}\right\rangle}(x)$, including the more common forms that involve a reordering of the vector $\left(f_{1}\left(x_{1}\right), f_{2}\left(x_{2}\right)\right.$, $\left.\ldots, f_{n}\left(x_{n}\right)\right)$.

Apparently, model $(S u)$ seems to be quite different from the noncompensatory sorting model. This is all the more true that it is often presented as requiring that all attributes are expressed on a common scale. The difference between the noncompensatory sorting model and model $(S u)$ is only apparent however.

Observe first that any partition that can be represented in the noncompensatory sorting model has a representation using a Sugeno integral. Indeed, let $\left\langle\mathscr{F},\left\langle\mathscr{A}_{i}\right\rangle_{i \in N}\right\rangle$ be a representation of $\langle\mathscr{A}, \mathscr{U}\rangle$ in the noncompensatory sorting model. For all $i \in N$, define $f_{i}$ letting, for all $x_{i} \in X_{i}$,

$$
\begin{cases}f_{i}\left(x_{i}\right)=1 & \text { if } x_{i} \in \mathscr{A}_{i} \\ f_{i}\left(x_{i}\right)=0 & \text { otherwise }\end{cases}
$$

and $\mu$ on $2^{N}$ letting, for all $A \in 2^{N}$,

$$
\begin{cases}\mu(A)=1 & \text { if } A \in \mathscr{F} \\ \mu(A)=0 & \text { otherwise. }\end{cases}
$$

With such definitions, we have, for all $x \in X$, either $S_{\left\langle\mu, f_{i}\right\rangle}(x)=1$ or $S_{\left\langle\mu, f_{i}\right\rangle}(x)=0$. We have $S_{\left\langle\mu, f_{i}\right\rangle}(x)=1$ iff $A(x)=\left\{i \in N: x_{i} \in \mathscr{A}_{i}\right\} \in \mathscr{F}$ iff $x \in \mathscr{A}$.

Consider now a partition $\langle\mathscr{A}, \mathscr{U}\rangle$ of $X$ that can be represented in model $(S u)$. Let us show that such a partition satisfies (13). Suppose that $\left(x_{i}, a_{-i}\right) \in$ $\mathscr{A},\left(y_{i}, b_{-i}\right) \in \mathscr{A}$ and $\left(x_{i}, b_{-i}\right) \notin \mathscr{A}$. Since $\left(y_{i}, b_{-i}\right) \in \mathscr{A},\left(x_{i}, b_{-i}\right) \notin \mathscr{A}$ implies, using the definition of model $(S u)$, that $f_{i}\left(x_{i}\right) \leq 0$. Since $\left(x_{i}, a_{-i}\right) \in \mathscr{A}$, we must have, for some $I \in 2^{N}$ such that $i \notin I, \mu(I)>0$ and $\bigwedge_{j \in I} f_{j}\left(a_{j}\right)>0$. This obviously implies $\left(z_{i}, a_{-i}\right) \in \mathscr{A}$, for all $z_{i} \in X_{i}$.

Combining the above observations and Lemma 20 with Theorem 21, we have proved: 


\section{Proposition 23}

A partition $\langle\mathscr{A}, \mathscr{U}\rangle$ of a set $X$ has a representation in the noncompensatory sorting model iff it has a representation in the Sugeno integral model (Su).

Słowiński et al. (2002, Theorem 2.4) state, without proof, that a partition $\langle\mathscr{A}, \mathscr{U}\rangle$ can be represented in the Sugeno integral model $(S u)$ iff it satisfies condition (13). The above proposition, connects Słowiński et al. (2002, Theorem 2.4) and our Theorem 21, showing, in fact, that they are characterizations of the same underlying model.

\section{Remark 24}

Clearly model $(S u)$, being equivalent to the noncompensatory sorting model, implies linearity. Hence, it can be represented in the decision rule model. Model $(S u)$ implies condition 2-graded which is not implied by the decision rule model. Hence, when model $(S u)$ is reinterpreted in the decision rule model, it must use rules that are more specific that the general "at least" rules used in the decision rule model. Greco et al. (2001b, Theorem 2.3, page 129) have investigated the type of rules that are generated by model $(S u)$, or, equivalently, by the noncompensatory sorting model. Although this gives another interpretation of the noncompensatory sorting model, this new interpretation is not crucial for our purposes and we refer the interested reader to Greco et al. (2001b) and Słowiński et al. (2002) for details.

\subsection{Extensions}

Unlike what happens with linear $i$, the roles of $\mathscr{A}$ and $\mathscr{U}$ are not symmetric

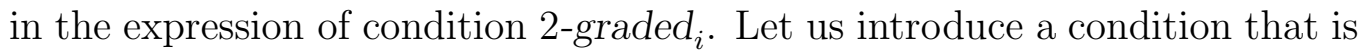
dual to condition 2 -graded $_{i}$ replacing $\mathscr{A}$ by $\mathscr{U}$. We say that $\langle\mathscr{A}, \mathscr{U}\rangle$ satisfies condition 2-graded ${ }_{i}^{*}$ if

$$
\left.\begin{array}{l}
\left(x_{i}, a_{-i}\right) \in \mathscr{U} \\
\text { and } \\
\left(y_{i}, a_{-i}\right) \in \mathscr{U} \\
\text { and } \\
\left(y_{i}, b_{-i}\right) \in \mathscr{U}
\end{array}\right\} \Rightarrow\left\{\begin{array}{c}
\left(x_{i}, b_{-i}\right) \in \mathscr{U} \\
\text { or } \\
\left(z_{i}, a_{-i}\right) \in \mathscr{U}
\end{array}\right.
$$

for all $x_{i}, y_{i}, z_{i} \in X_{i}$ and all $a_{-i}, b_{-i} \in X_{-i}$. We say that $\langle\mathscr{A}, \mathscr{U}\rangle$ is 2 -graded* if it satisfies 2-graded ${ }_{i}^{*}$ on all $i \in N$.

Condition 2-graded ${ }_{i}^{*}$ is implied by the noncompensatory sorting model. Indeed, suppose that 2 -graded ${ }_{i}^{*}$ is violated so that, for some $x_{i}, y_{i}, z_{i} \in X_{i}$ and some $a_{-i}, b_{-i} \in X_{-i},\left(x_{i}, a_{-i}\right) \in \mathscr{U},\left(y_{i}, a_{-i}\right) \in \mathscr{U},\left(y_{i}, b_{-i}\right) \in \mathscr{U}$, $\left(x_{i}, b_{-i}\right) \in \mathscr{A}$ and $\left(z_{i}, a_{-i}\right) \in \mathscr{A}$. This implies $\operatorname{Not}\left[x_{i} \succsim_{i} z_{i}\right], \operatorname{Not}\left[y_{i} \succsim_{i} z_{i}\right]$ and $\operatorname{Not}\left[y_{i} \succsim_{i} x_{i}\right]$, violating the fact that $\succsim_{i}$ has at most two distinct equivalence classes in the noncompensatory sorting model. We have: 


\section{Lemma 25}

1. Conditions 2-graded $i$ and 2-graded ${ }_{i}^{*}$ are independent,

2. In presence of linear $i$, conditions $2-\operatorname{graded}_{i}$ and 2-graded ${ }_{i}^{*}$ are equivalent,

3. $\left[2-\operatorname{graded}_{i}\right.$ and $\left.2-\operatorname{graded}_{i}^{*}\right]$ do not imply linear .

ProOF

Part 1. We give below the example of a partition $\langle\mathscr{A}, \mathscr{U}\rangle$ that is 2 -graded and satisfies condition 2-graded ${ }_{i}^{*}$ on all but one attribute. Exchanging the roles of $\mathscr{A}$ and $\mathscr{U}$ gives the other required example.

\section{Example 26 (2-graded and Not[2-graded $\left.\left.{ }_{i}^{*}\right]\right)$}

Let $n=2$ and $X=\left\{x_{1}, y_{1}, z_{1}\right\} \times\left\{x_{2}, y_{2}\right\}$. Let $\mathscr{A}=\left\{\left(x_{1}, x_{2}\right),\left(y_{1}, y_{2}\right)\right\}$. Conditions 2-graded 2 and 2-graded 2 hold since $X_{2}$ has only two elements. Condition 2-graded 1 is trivially satisfied. Condition 2-graded ${ }_{1}^{*}$ is violated since $\left(y_{1}, x_{2}\right) \in \mathscr{U},\left(z_{1}, x_{2}\right) \in \mathscr{U}$ and $\left(z_{1}, y_{2}\right) \in \mathscr{U}$ but $\left(y_{1}, y_{2}\right) \in \mathscr{A}$ and $\left(x_{1}, x_{2}\right) \in \mathscr{A}$.

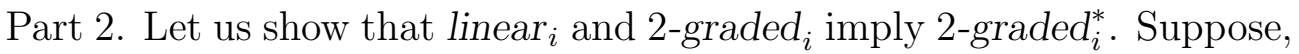
in violation of 2 -graded ${ }_{i}^{*}$, that $\left(x_{i}, a_{-i}\right) \in \mathscr{U},\left(y_{i}, a_{-i}\right) \in \mathscr{U},\left(y_{i}, b_{-i}\right) \in \mathscr{U}$, $\left(x_{i}, b_{-i}\right) \in \mathscr{A}$ and $\left(z_{i}, a_{-i}\right) \in \mathscr{A}$. Using linear $,\left(x_{i}, b_{-i}\right) \in \mathscr{A},\left(z_{i}, a_{-i}\right) \in \mathscr{A}$ and $\left(x_{i}, a_{-i}\right) \in \mathscr{U}$ imply $\left(z_{i}, b_{-i}\right) \in \mathscr{A}$. Using 2-graded $i,\left(x_{i}, b_{-i}\right) \in \mathscr{A}$, $\left(z_{i}, b_{-i}\right) \in \mathscr{A}$ and $\left(z_{i}, a_{-i}\right) \in \mathscr{A}$ imply either $\left(x_{i}, a_{-i}\right) \in \mathscr{A}$ or $\left(y_{i}, b_{-i}\right) \in \mathscr{A}$, a contradiction.

Conversely, suppose that linear $_{i}$ and 2 -graded $_{i}^{*}$ hold. Suppose, in violation of 2 -graded $_{i}$ that $\left(x_{i}, a_{-i}\right) \in \mathscr{A},\left(y_{i}, a_{-i}\right) \in \mathscr{A},\left(y_{i}, b_{-i}\right) \in \mathscr{A},\left(x_{i}, b_{-i}\right) \in$ $\mathscr{U}$ and $\left(z_{i}, a_{-i}\right) \in \mathscr{U}$. Suppose that $\left(z_{i}, b_{-i}\right) \in \mathscr{U}$. Using 2-graded ${ }_{i}^{*}$, $\left(x_{i}, b_{-i}\right) \in \mathscr{U},\left(z_{i}, b_{-i}\right) \in \mathscr{U}$ and $\left(z_{i}, a_{-i}\right) \in \mathscr{U}$ imply $\left(x_{i}, a_{-i}\right) \in \mathscr{U}$ or $\left(y_{i}, a_{-i}\right) \in \mathscr{U}$, a contradiction. Hence we must have $\left(z_{i}, b_{-i}\right) \in \mathscr{A}$. Using linear $_{i},\left(x_{i}, a_{-i}\right) \in \mathscr{A}$ and $\left(z_{i}, b_{-i}\right) \in \mathscr{A}$ imply $\left(z_{i}, a_{-i}\right) \in \mathscr{A}$ or $\left(x_{i}, b_{-i}\right) \in \mathscr{A}$, a contradiction.

Part 3. Since when each $X_{i}$ has only two elements, 2-graded ${ }_{i}$ and 2-graded ${ }_{i}^{*}$ are trivially satisfied, Example 18 above shows that linear ${ }_{i}$ may fail on an attribute while 2-graded $i$ and 2-graded ${ }_{i}^{*}$ hold and all $i \in N$.

This shows that we can replace condition 2-graded by 2-graded* in the characterization of the noncompensatory sorting model proposed in Theorem 21. Therefore the asymmetry of the roles of $\mathscr{A}$ and $\mathscr{U}$ in the statement of Theorem 21 is only apparent. Furthermore, the conjunction of 2 -graded $_{i}$ and 2 -graded ${ }_{i}^{*}$ without supposing linear $_{i}$ has interesting consequences as noted in the following: 


\section{Lemma 27}

$\langle\mathscr{A}, \mathscr{U}\rangle$ satisfies 2 -graded $_{i}$ and 2 -graded $_{i}^{*}$ iff $\sim_{i}$ has at most two equivalence classes.

PROOF

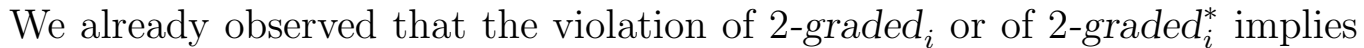
that $\sim_{i}$ has at least three distinct equivalence classes. Let us show sufficiency.

Suppose that $\left(x_{i}, a_{-i}\right) \in \mathscr{A}$ and $\left(y_{i}, a_{-i}\right) \in \mathscr{U}$, so that $x_{i}$ and $y_{i}$ belong to distinct equivalence classes of $\sim_{i}$. Suppose that $\left(z_{i}, b_{-i}\right) \in \mathscr{A}$ and $\left(y_{i}, b_{-i}\right) \in$ $\mathscr{U}$, the case in which $\left(y_{i}, b_{-i}\right) \in \mathscr{A}$ and $\left(z_{i}, b_{-i}\right) \in \mathscr{U}$ being dealt with similarly.

We must show that $x_{i}$ and $z_{i}$ belong to the same equivalence class of $\sim_{i}$, i.e., that $\left(x_{i}, c_{-i}\right) \in \mathscr{A} \Leftrightarrow\left(z_{i}, c_{-i}\right) \in \mathscr{A}$. Let us first observe that we cannot have $\left(z_{i}, a_{-i}\right) \in \mathscr{U}$. Indeed, using 2 -graded ${ }_{i}^{*},\left(z_{i}, a_{-i}\right) \in \mathscr{U},\left(y_{i}, a_{-i}\right) \in \mathscr{U}$ and $\left(y_{i}, b_{-i}\right) \in \mathscr{U}$ would imply $\left(z_{i}, b_{-i}\right) \in \mathscr{U}$ or $\left(x_{i}, a_{-i}\right) \in \mathscr{U}$, a contradiction. Hence, we have $\left(z_{i}, a_{-i}\right) \in \mathscr{A}$.

Suppose that $\left(x_{i}, c_{-i}\right) \in \mathscr{A}$. Using 2 -graded $i,\left(z_{i}, a_{-i}\right) \in \mathscr{A},\left(x_{i}, a_{-i}\right) \in \mathscr{A}$ and $\left(x_{i}, c_{-i}\right) \in \mathscr{A}$ imply $\left(z_{i}, c_{-i}\right) \in \mathscr{A}$ or $\left(y_{i}, a_{-i}\right) \in \mathscr{A}$. Hence, we must have $\left(z_{i}, c_{-i}\right) \in \mathscr{A}$.

Conversely, suppose that $\left(z_{i}, c_{-i}\right) \in \mathscr{A}$. Using 2-graded,$\left(x_{i}, a_{-i}\right) \in \mathscr{A}$, $\left(z_{i}, a_{-i}\right) \in \mathscr{A}$ and $\left(z_{i}, c_{-i}\right) \in \mathscr{A}$ imply $\left(x_{i}, c_{-i}\right) \in \mathscr{A}$ or $\left(y_{i}, a_{-i}\right) \in \mathscr{A}$. Hence, we must have $\left(x_{i}, c_{-i}\right) \in \mathscr{A}$.

Consider a model such that:

- for all $i \in N$, there is a set $\mathscr{A}_{i} \subseteq X_{i}$,

- there is a subset $\mathscr{F}$ of $2^{N}$,

such that, for all $x \in X$,

$$
x \in \mathscr{A} \Leftrightarrow\left\{i \in N: x_{i} \in \mathscr{A}_{i}\right\} \in \mathscr{F} .
$$

The only difference between this model and the noncompensatory sorting model is that $\mathscr{F}$ is no longer supposed to satisfy (11). We call this model the generalized noncompensatory sorting model. This model is nothing but the particular case of model $(D 0)$ in which each function $u_{i}$ can take at most two distinct values.

In such a model, each relation $\sim_{i}$ has at most two distinct equivalence classes, so that it implies conditions 2-graded and 2-graded*. The following shows that the converse is true.

\section{Proposition 28}

A partition $\langle\mathscr{A}, \mathscr{U}\rangle$ has a representation in the generalized noncompensatory sorting model iff it is 2-graded and 2-graded*. 


\section{PROOF}

Necessity is obvious. We show sufficiency. Let $i \in N$. We know from lemma 27 that $\sim_{i}$ has at most two equivalence classes. If $\sim_{i}$ has only one equivalence class, let $\mathscr{A}_{i}=X_{i}$. If $\sim_{i}$ has two distinct equivalence classes, let $\mathscr{A}_{i}$ be any of them. Define $\mathscr{F}$ letting $I \in \mathscr{F}$ whenever there is some $x \in \mathscr{A}$ such that $A(x)=I$.

By construction, $x \in \mathscr{A}$ implies $A(x) \in \mathscr{F}$. Conversely, suppose that $A(x) \in \mathscr{F}$. By construction, this implies that for some $y \in X$, we have $y \in \mathscr{A}$ and $A(y)=A(x)$. That $x \in \mathscr{A}$ follows from the definition of the sets $\mathscr{A}_{i}$ and Part 2 of lemma 1.

\section{Remark 29}

As already mentioned, it is tempting to interpret $\mathscr{A}$ as the set of satisfactory alternatives in the noncompensatory sorting model (although its interpretation as the set of unsatisfactory alternatives is also possible in view of the symmetric roles of $\mathscr{A}$ and $\mathscr{U}$ in the model). With this interpretation in mind, we may interpret the sets $\mathscr{A}_{i}$ as the set of "satisfactory levels" on $X_{i}$. The fact that $\mathscr{F}$ satisfies (11) simply means that replacing an unsatisfactory level, i.e., a level in $\mathscr{U}_{i}$, by a satisfactory level, i.e., a level in $\mathscr{A}_{i}$, cannot turn a satisfactory alternative into an unsatisfactory one. Note however that this is not the only possible interpretation.

An alternative interpretation of the noncompensatory sorting model is that $\mathscr{A}$ and $\mathscr{U}$ are not ordered in terms of desirability. In this case, the sets $\mathscr{A}_{i}$ can be interpreted as a set of levels that are "typical" of category $\mathscr{A}$. The fact that $\mathscr{F}$ satisfies (11) means here modifying the evaluations of an object in $\mathscr{A}$ replacing non-typical levels by typical ones will keep this object in $\mathscr{A}$.

Such an interpretation is no longer warranted in the generalized noncompensatory sorting model. Indeed, the levels in $\mathscr{A}_{i}$ cannot be considered as typical of $\mathscr{A}$. What is now typical of $\mathscr{A}$ are particular combinations of levels in $\mathscr{A}_{i}$. Suppose, e.g., that you sort cars evaluated on two attributes into two categories: "attractive" and "ugly". It may well be possible that you would find attractive a car that is either [red and sporty] or [dark and luxurious], while you would reject as ugly a [dark and sporty] or a [red and luxurious] one (think, e.g., of two well-known Italian and German car-makers). Being red or dark is not here typical of being attractive or ugly; being [red and sporty] or [dark and luxurious] is. This would be impossible in the noncompensatory sorting model while this is clearly compatible with the generalized noncompensatory sorting model.

We leave to the reader the easy interpretation of such a model in terms of decision rules (clearly this interpretation will use decision rules that are not all of the "at least" type since the generalized noncompensatory sorting model does not imply linearity). 


\subsection{Uniqueness and degenerate attributes}

We have already observed that, when all attributes are influent, the representation of $\langle\mathscr{A}, \mathscr{U}\rangle$ in the noncompensatory sorting model is unique. In this case, each relation $\succsim_{i}$ has exactly two distinct equivalence classes and $\mathscr{A}_{i}$ always coincides with the elements in the first equivalence class of $\succsim_{i}$. Let us now show that this is indeed a necessary condition for the uniqueness of the representation in the noncompensatory sorting model. Indeed, suppose that $j \in N$ is degenerate. In the proof of Theorem 21 and Proposition 16 (see also Remark 22), we have built a representation such that $\mathscr{A}_{j}=\varnothing$. This implies that there is no $x \in X$ such that $j \in A(x)$. In order to guarantee that the set $\mathscr{F}$ satisfies (11), we have built it in such a way that $I \in \mathscr{F}$ if $A(x) \subseteq I$ for some $x \in \mathscr{A}$. Keeping the same set $\mathscr{F}$, we can freely choose $\mathscr{A}_{j}$ to be an arbitrary subset of $X_{j}$. This shows that the representation of $\langle\mathscr{A}, \mathscr{U}\rangle$ in the noncompensatory sorting model is unique if and only if all attributes are influent for $\langle\mathscr{A}, \mathscr{U}\rangle$. Note that, if on each degenerate attribute $j \in N$, we modify the sets $\mathscr{A}_{j}$ taking them to be an arbitrary strict subset of $X_{i}$, we will still have that $I \in \mathscr{F}$ if $A(x) \subseteq I$ for some $x \in \mathscr{A}$. If, furthermore, all the modified sets are nonempty, for all $I \in 2^{N}$, we have $A(x)=I$, for some $x \in X$. The non-uniqueness of the representation in the noncompensatory sorting model in presence of degenerate attributes is illustrated below.

\section{Example 30 (Impact of degenerate attributes)}

Let $n=3$ and $X_{1}=X_{2}=X_{3}=\{9,10,11\}$. Let $\mathscr{A}=\{(10,10,9)$, $(10,10,10),(10,10,11),(10,11,9),(10,11,10),(10,11,11),(11,10,9),(11$, 10,10), $(11,10,11),(11,11,9),(11,11,10),(11,11,11)\}$ and $\mathscr{U}=X \backslash \mathscr{A}$. This partition $\langle\mathscr{A}, \mathscr{U}\rangle$ can be obtained using the pessimistic ELECTRE TRI model with, using the same notation as above, $p=(10,10,10), V_{i}=\varnothing$ and $S_{i}=\geq$ for all $i \in N, w_{1}=w_{2}=0.4, w_{3}=0.2$ and $\lambda=2 / 3$. This shows that it is linear and 2-graded.

It is apparent that attributes 1 and 2 are influent while attribute 3 is not. The representation built in Theorem 21 is such that $\mathscr{A}_{1}=\mathscr{A}_{2}=\{10,11\}$, $\mathscr{A}_{3}=\varnothing$ and $\mathscr{F}=\{\{1,2\},\{1,2,3\}\}$. Keeping the same $\mathscr{F}$, we can modify $\mathscr{A}_{3}$ taking it to be an arbitrary subset of $X_{3}$, e.g., $\{9\},\{11\}$ or $\{9,11\}$. This is detailed in Table 2 .

\section{Remark 31}

Consider a partition $\langle\mathscr{A}, \mathscr{U}\rangle$ that has a representation $\left\langle\mathscr{F},\left\langle\mathscr{A}_{i}\right\rangle_{i \in N}\right\rangle$ in the noncompensatory sorting model. If $i \in N$ is influent for $\langle\mathscr{A}, \mathscr{U}\rangle$, we know that $\succsim_{i}$ is a weak order having exactly two equivalence classes and $\mathscr{A}_{i}$ coincides with the first equivalence class of $\succsim_{i}$. If $i \in N$ is degenerate for $\langle\mathscr{A}, \mathscr{U}\rangle$, $\succsim_{i}$ is a weak order having just one equivalence class. We have taken $\mathscr{A}_{i}$ to 


\begin{tabular}{cccccccc}
$x$ & Cat. & $A(x)$ & $A^{\prime}(x)$ & $x$ & Cat. & $A(x)$ & $A^{\prime}(x)$ \\
\hline$(10,10,9)$ & $\mathscr{A}$ & $\{1,2\}$ & $\{1,2\}$ & $(9,9,9)$ & $\mathscr{U}$ & $\varnothing$ & $\varnothing$ \\
$(10,10,10)$ & $\mathscr{A}$ & $\{1,2\}$ & $\{1,2\}$ & $(9,9,10)$ & $\mathscr{U}$ & $\varnothing$ & $\varnothing$ \\
$(10,10,11)$ & $\mathscr{A}$ & $\{1,2\}$ & $\{1,2,3\}$ & $(9,9,11)$ & $\mathscr{U}$ & $\varnothing$ & $\{3\}$ \\
$(10,11,9)$ & $\mathscr{A}$ & $\{1,2\}$ & $\{1,2\}$ & $(9,10,9)$ & $\mathscr{U}$ & $\{2\}$ & $\{2\}$ \\
$(10,11,10)$ & $\mathscr{A}$ & $\{1,2\}$ & $\{1,2\}$ & $(9,10,10)$ & $\mathscr{U}$ & $\{2\}$ & $\{2\}$ \\
$(10,11,11)$ & $\mathscr{A}$ & $\{1,2\}$ & $\{1,2,3\}$ & $(9,10,11)$ & $\mathscr{U}$ & $\{2\}$ & $\{2,3\}$ \\
$(11,10,9)$ & $\mathscr{A}$ & $\{1,2\}$ & $\{1,2\}$ & $(9,11,9)$ & $\mathscr{U}$ & $\{2\}$ & $\{2\}$ \\
$(11,10,10)$ & $\mathscr{A}$ & $\{1,2\}$ & $\{1,2\}$ & $(9,11,10)$ & $\mathscr{U}$ & $\{2\}$ & $\{2\}$ \\
$(11,9,10)$ & $\mathscr{A}$ & $\{1,2\}$ & $\{1,2\}$ & $(9,11,11)$ & $\mathscr{U}$ & $\{2\}$ & $\{2,3\}$ \\
$(11,10,11)$ & $\mathscr{A}$ & $\{1,2\}$ & $\{1,2,3\}$ & $(10,9,9)$ & $\mathscr{U}$ & $\{1\}$ & $\{1\}$ \\
$(11,11,9)$ & $\mathscr{A}$ & $\{1,2\}$ & $\{1,2\}$ & $(10,9,10)$ & $\mathscr{U}$ & $\{1\}$ & $\{1\}$ \\
$(11,11,10)$ & $\mathscr{A}$ & $\{1,2\}$ & $\{1,2\}$ & $(10,9,11)$ & $\mathscr{U}$ & $\{1\}$ & $\{1,3\}$ \\
$(11,11,11)$ & $\mathscr{A}$ & $\{1,2\}$ & $\{1,2,3\}$ & $(11,9,9)$ & $\mathscr{U}$ & $\{1\}$ & $\{1\}$ \\
& & & & $(11,9,10)$ & $\mathscr{U}$ & $\{1\}$ & $\{1\}$ \\
& & & & $(11,9,11)$ & $\mathscr{U}$ & $\{1\}$ & $\{1,3\}$
\end{tabular}

Table 2: Details of Example 30.

Attributes 1 and 2 are influent. Attribute 3 is degenerate.

$$
\begin{gathered}
\mathscr{A}_{1}=\mathscr{A}_{2}=\{10,11\}, \mathscr{A}_{3}=\varnothing, \\
\mathscr{A}_{1}^{\prime}=\mathscr{A}_{2}^{\prime}=\{10,11\}, \mathscr{A}_{3}^{\prime}=\{11\}, \\
\mathscr{F}=\{\{1,2\},\{1,2,3\}\}, \\
A(x)=\left\{i \in N: x_{i} \in \mathscr{A}_{i}\right\}, A^{\prime}(x)=\left\{i \in N: x_{i} \in \mathscr{A}_{i}^{\prime}\right\} .
\end{gathered}
$$

be empty in this case but it can be freely modified, keeping the same set $\mathscr{F}$, to be an arbitrary subset of $X_{i}$. Modify the representation of $\langle\mathscr{A}, \mathscr{U}\rangle$ in the noncompensatory sorting model, taking, on all attributes $i \in N$ that are degenerate $\mathscr{A}_{i}=X_{i}$. Take $S_{i}=\succsim_{i}$, for all $i \in N$ and take $p_{i}$ to be an arbitrary element in the first equivalence class of $\succsim_{i}$. We have, for all $i \in N$, $x_{i} S_{i} p_{i}$ iff $x_{i} \in \mathscr{A}_{i}$, so that

$$
x \in \mathscr{A} \Leftrightarrow A(x) \in \mathscr{F} \Leftrightarrow S(x, p) \in \mathscr{F}
$$

Observe that if the set $\mathscr{F}$ has a joint additive representation à la ELECTRE, i.e., that, for all $i \in N$, there are nonnegative weights $w_{i}$ and a real number $\lambda$ between $1 / 2$ and 1 such that, for all $I \subseteq 2^{N}$,

$$
I \in \mathscr{F} \Leftrightarrow \sum_{i \in I} w_{i} \geq \lambda,
$$

the above construction is exactly equivalent to the concordance part of ELECTRE TRI.

Neglecting the question of the additive representation of the set $\mathscr{F}$, this shows that a twofold partition that can be obtained in ELECTRE TRI (with $V_{i}=\varnothing$, for all $i \in N$ ) using a profile that is outside the set $X$ can always 
be obtained with ELECTRE TRI (still with $V_{i}=\varnothing$, for all $i \in N$ ) using a profile that belongs to $X$.

\subsection{Conjunctive and disjunctive sorting models}

We say $\langle\mathscr{A}, \mathscr{U}\rangle$ satisfies condition conj if

$$
\left.\begin{array}{l}
\left(x_{i}, a_{-i}\right) \in \mathscr{A} \\
\text { and } \\
\left(y_{i}, a_{-i}\right) \in \mathscr{A} \\
\text { and } \\
\left(y_{i}, b_{-i}\right) \in \mathscr{A}
\end{array}\right\} \Rightarrow\left(x_{i}, b_{-i}\right) \in \mathscr{A}
$$

for all $i \in N$, all $x_{i}, y_{i}, z_{i} \in X_{i}$ and all $a_{-i}, b_{-i} \in X_{-i}$. Condition conj is obviously necessary for the conjunctive sorting model. It is obvious that it implies that $\langle\mathscr{A}, \mathscr{U}\rangle$ is 2 -graded. It is easy to build examples showing that linearity and conj are independent conditions. We omit the simple proof of the following:

\section{Proposition 32}

A partition $\langle\mathscr{A}, \mathscr{U}\rangle$ has a representation in the conjunctive sorting model iff it is linear and satisfies conj.

Noting the duality between the conjunctive and the disjunctive sorting models, it is easy to devise a similar proposition for the case of the disjunctive sorting model. We leave the details to the interested reader. Let us finally observe that, within the decision rule model, the conjunctive and disjunctive models have a particularly simple interpretation since they use "at least" decision rules having a very elementary syntax.

\section{The noncompensatory sorting model with veto for twofold partitions}

\subsection{Definitions}

Let $\langle\mathscr{A}, \mathscr{U}\rangle$ be a twofold partition of $X$. We say that $\langle\mathscr{A}, \mathscr{U}\rangle$ has a representation in the noncompensatory sorting model with veto if:

- for all $i \in N$, there are disjoint sets $\mathscr{A}_{i}, \mathscr{V}_{i} \subseteq X_{i}$,

- there is a subset $\mathscr{F}$ of $2^{N}$ satisfying (11), 
such that, for all $x \in X$,

$$
x \in \mathscr{A} \Leftrightarrow\left[\left\{i \in N: x_{i} \in \mathscr{A}_{i}\right\} \in \mathscr{F} \text { and }\left\{i \in N: x_{i} \in \mathscr{V}_{i}\right\}=\varnothing\right] .
$$

In this case, we say, that $\left\langle\mathscr{F},\left\langle\mathscr{A}_{i}, \mathscr{V}_{i}\right\rangle_{i \in N}\right\rangle$ is a representation of $\langle\mathscr{A}, \mathscr{U}\rangle$ in the noncompensatory sorting model with veto. We write $A(x)$ and $V(x)$ instead of $\left\{i \in N: x_{i} \in \mathscr{A}_{i}\right\}$ and $\left\{i \in N: x_{i} \in \mathscr{V}_{i}\right\}$ when there is no risk of confusion on the underlying sets $\mathscr{A}_{i}$ and $\mathscr{V}_{i}$. We define, in this section, $\mathscr{U}_{i}$ as $X_{i} \backslash\left[\mathscr{A}_{i} \cup \mathscr{V}_{i}\right]$

The noncompensatory sorting model is clearly a particular case of the noncompensatory sorting model with veto. The interpretation of the noncompensatory sorting model with veto is similar to the one of the noncompensatory sorting model considered in the preceding section. The only difference here is that there may exist a subset $\mathscr{V}_{i}$ of elements of $X_{i}$ that are "repulsive" for $\mathscr{A}$ in that, as soon as one of the evaluations of $x \in X$ is repulsive, it is impossible to have $x \in \mathscr{A}$. Note that, with the presence of repulsive levels for $\mathscr{A}$, the roles of $\mathscr{A}$ and $\mathscr{U}$ are no longer symmetric in the noncompensatory sorting model with veto.

The pessimistic version of ELECTRE TRI, when preference and indifference thresholds are equal, is a particular case of the noncompensatory sorting model with veto. Indeed, using the notation of Section 4, we have, for all $x \in X$,

$$
x \in \mathscr{A} \Leftrightarrow x S p \Leftrightarrow\left[\sum_{i \in S(x, p)} w_{i} \geq \lambda \text { and }\left[\operatorname{Not}\left[p_{i} V_{i} x_{i}\right], \text { for all } i \in N\right]\right] \text {. }
$$

Defining $\mathscr{A}_{i}=\left\{x_{i} \in X_{i}: x_{i} S_{i} p_{i}\right\}, \mathscr{V}_{i}=\left\{x_{i} \in X_{i}: p_{i} V_{i} x_{i}\right\}$ and letting $I \in \mathscr{F}$ if and only if $\sum_{i \in I} w_{i} \geq \lambda$, shows that such a model is a particular case of the noncompensatory sorting model with veto. Note that the sets $\mathscr{A}_{i}$ and $\mathscr{V}_{i}$ are indeed disjoint because we have supposed that $V_{i}$ is included in the asymmetric part of $S_{i}$ : if $x_{i} S_{i} p_{i}$, we cannot have $p_{i} V_{i} x_{i}$.

\subsection{Axioms and results}

Let us first observe that if a partition $\langle\mathscr{A}, \mathscr{U}\rangle$ has a representation in the noncompensatory sorting model with veto then it must be linear.

\section{Lemma 33}

If $\langle\mathscr{A}, \mathscr{U}\rangle$ has a representation in the noncompensatory sorting model with veto then it is linear. 


\section{Proof}

Suppose that $\left(x_{i}, a_{-i}\right) \in \mathscr{A}$ and $\left(y_{i}, b_{-i}\right) \in \mathscr{A}$. By construction, we know that $x_{i}$ and $y_{i}$ cannot belong to $\mathscr{V}_{i}$. If $x_{i} \in \mathscr{A}_{i},\left(y_{i}, b_{-i}\right) \in \mathscr{A}$, using (11) implies $\left(x_{i}, b_{-i}\right) \in \mathscr{A}$. If $x_{i} \in \mathscr{U}_{i}$, since $\left(x_{i}, a_{-i}\right) \in \mathscr{A}$ and $y_{i} \in \mathscr{A}_{i} \cup \mathscr{U}_{i}$, using (11) implies $\left(y_{i}, a_{-i}\right) \in \mathscr{A}$.

It remains to see what must be added to linearity in order to characterize the noncompensatory sorting model with veto. Again, this will require limiting the number of distinct equivalence classes of $\succsim_{i}$, this time taking into account the possible existence of an equivalence class corresponding to repulsive levels for $\mathscr{A}$.

We say that the partition $\langle\mathscr{A}, \mathscr{U}\rangle$ is 3 -graded with veto on attribute $i \in N$ $\left(\right.$ condition $\left.3 v-\operatorname{graded}_{i}\right)$ if, for all $x_{i}, y_{i}, z_{i} \in X_{i}$ and all $a_{-i}, b_{-i}, c_{i} \in X_{-i}$,

$$
\left.\begin{array}{l}
\left(x_{i}, a_{-i}\right) \in \mathscr{A} \\
\text { and } \\
\left(y_{i}, a_{-i}\right) \in \mathscr{A} \\
\text { and } \\
\left(y_{i}, b_{-i}\right) \in \mathscr{A} \\
\text { and } \\
\left(z_{i}, c_{-i}\right) \in \mathscr{A}
\end{array}\right\} \Rightarrow\left\{\begin{array}{c}
\left(x_{i}, b_{-i}\right) \in \mathscr{A} \\
\text { or } \\
\left(z_{i}, a_{-i}\right) \in \mathscr{A}
\end{array}\right.
$$

$\langle\mathscr{A}, \mathscr{U}\rangle$ is said to be 3-graded with veto if it satisfies $3 \mathrm{v}$-graded gror $_{i}$ all $i \in N$. This condition is inspired by Greco et al. (2001a) who study veto effects in the context of binary relations. It is apparent that condition 2-graded implies condition $3 \mathrm{v}$-graded $i$. The role of condition $3 \mathrm{v}$-graded $i$ is to limit the number of distinct equivalence classes of $\succsim_{i}$, taking into account the possible existence of repulsive levels. The intuition behind this condition is simple. The premises of the condition imply that none of $x_{i}, y_{i}$ and $z_{i}$ can be repulsive for $\mathscr{A}$. Suppose that linear $_{i}$ holds and that $\operatorname{Not}\left[\left(x_{i}, b_{-i}\right) \in \mathscr{A}\right]$. Since $\left(y_{i}, b_{-i}\right) \in \mathscr{A}$ this implies $y_{i} \succ_{i} x_{i}$. If the relation $\succsim_{i}$ only has at most three equivalence classes with the last class containing repulsive levels, this implies that, for all $z_{i} \in X_{i}$ that are not repulsive for $\mathscr{A}$, it must be true that $z_{i} \succsim_{i} x_{i}$. Therefore if $\left(x_{i}, a_{-i}\right) \in \mathscr{A}$, we must have $\left(z_{i}, a_{-i}\right) \in \mathscr{A}$, as required by condition $3 \mathrm{v}-\operatorname{graded}_{i}$. The role of the additional premise $\left(y_{i}, a_{-i}\right) \in \mathscr{A}$ is to ensure that condition $3 \mathrm{v}$-graded $i$ is independent from linear ${ }_{i}$. We have:

\section{Lemma 34}

1. If $\langle\mathscr{A}, \mathscr{U}\rangle$ has a representation in the noncompensatory sorting model with veto then it is 3-graded with veto.

2. Conditions linear $i$ and $3 \mathrm{v}$-graded $i$ are independent. 


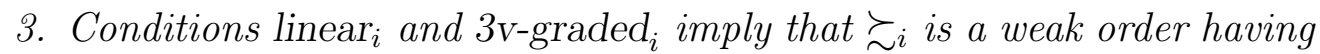
at most three equivalence classes.

Furthermore if $\succsim_{i}$ has exactly three distinct equivalence classes and if $x_{i}$ belongs to the last equivalence class of $\succsim_{i}$ then $\left(x_{i}, a_{-i}\right) \in \mathscr{U}$, for all $a_{-i} \in X_{-i}$.

\section{ProOF}

Part 1. Suppose that $\left(x_{i}, a_{-i}\right) \in \mathscr{A},\left(y_{i}, a_{-i}\right) \in \mathscr{A},\left(y_{i}, b_{-i}\right) \in \mathscr{A}$ and $\left(z_{i}, c_{-i}\right) \in \mathscr{A}$. By construction, none of $x_{i}, y_{i}$ and $z_{i}$ can be in $\mathscr{V}_{i}$. If $\left(x_{i}, b_{-i}\right) \notin \mathscr{A},\left(y_{i}, b_{-i}\right) \in \mathscr{A}$ implies that $x_{i} \in \mathscr{U}_{i}$ and $y_{i} \in \mathscr{A}_{i}$. Since $\left(x_{i}, a_{-i}\right) \in \mathscr{A}$ and $x_{i} \in \mathscr{U}_{i}$, using (11) implies that, for all $z_{i} \in X_{i} \backslash \mathscr{N}_{i}$, we have $\left(z_{i}, a_{-i}\right) \in \mathscr{A}$. Hence, condition $3 v$-graded grolds. $_{i}$.

Part 2. Using an additive model for sorting, it is easy to build partitions that are linear and satisfy $3 \mathrm{v}$-graded $i$ on all but one attribute. Observe that, when $X_{i}$ has only two elements, condition $3 \mathrm{v}$-graded $i$ trivially holds. Therefore, Example 18 above gives an example of a partition that is 3 -graded with veto and satisfies linear ${ }_{i}$ on all but one attribute.

Part 3. Since linear $_{i}$ holds, we know that $\succsim_{i}$ is complete. Suppose that there are $x_{i}, y_{i}, z_{i}, w_{i} \in X_{i}$ such that $x_{i} \succ_{i} y_{i}, y_{i} \succ_{i} z_{i}$ and $z_{i} \succ_{i} w_{i}$. By definition, this implies that, for some $a_{-i}, b_{-i}, c_{-i} \in X_{-i},\left(x_{i}, a_{-i}\right) \in \mathscr{A}$, $\left(y_{i}, a_{-i}\right) \notin \mathscr{A},\left(y_{i}, b_{-i}\right) \in \mathscr{A},\left(z_{i}, b_{-i}\right) \notin \mathscr{A}$ and $\left(z_{i}, c_{-i}\right) \in \mathscr{A},\left(w_{i}, c_{-i}\right) \notin \mathscr{A}$.

Using linear $i,\left(x_{i}, a_{-i}\right) \in \mathscr{A},\left(y_{i}, b_{-i}\right) \in \mathscr{A}$ and $\left(y_{i}, a_{-i}\right) \notin \mathscr{A}$, imply $\left(x_{i}, b_{-i}\right) \in \mathscr{A}$. Using $3 v-\operatorname{graded}_{i},\left(y_{i}, b_{-i}\right) \in \mathscr{A},\left(x_{i}, b_{-i}\right) \in \mathscr{A},\left(x_{i}, a_{-i}\right) \in \mathscr{A}$ and $\left(z_{i}, c_{-i}\right) \in \mathscr{A}$ imply $\left(y_{i}, a_{-i}\right) \in \mathscr{A}$ or $\left(z_{i}, b_{-i}\right) \in \mathscr{A}$, a contradiction.

Observe that the above proof shows that, when $\succsim_{i}$ has three distinct equivalence classes, then, for all $z_{i}$ in the last equivalence class, it is impossible to have $\left(z_{i}, c_{-i}\right) \in \mathscr{A}$.

The main result of this paper says that linearity and 3-gradedness with veto characterize the noncompensatory sorting model with veto.

\section{Theorem 35}

A partition $\langle\mathscr{A}, \mathscr{U}\rangle$ is representable in the noncompensatory sorting model with veto iff it is linear and 3-graded with veto.

\section{PROOF}

Necessity results from Lemmas 33 and 34. We show sufficiency.

Let $Z_{i}=\left\{x_{i} \in X_{i}:\left(x_{i}, a_{-i}\right) \in \mathscr{U}\right.$, for all $\left.a_{-i} \in X_{-i}\right\}$. Take $\mathscr{V}_{i}=Z_{i}$ and let $Y_{i}=X_{i} \backslash Z_{i}$. The sets $\mathscr{A}_{i}$ will all be subsets of $Y_{i}$ so that $\mathscr{V}_{i}$ and $\mathscr{A}_{i}$ will be disjoint. Consider the set $Y=\prod_{i=1}^{n} Y_{i}$ and let $\mathscr{A}^{\prime}=\mathscr{A} \cap Y$ and $\mathscr{U}^{\prime}=\mathscr{U} \cap Y$. Since $\langle\mathscr{A}, \mathscr{U}\rangle$ is a partition, we have $\mathscr{A} \neq \varnothing$ so that $Y$ cannot be empty. 
Suppose first that all attributes are degenerate for $\left\langle\mathscr{A}^{\prime}, \mathscr{U}^{\prime}\right\rangle$. By construction of the set $Y$, we must have $\mathscr{A}^{\prime}=Y$. In this case, take $\mathscr{A}_{i}=Y_{i}$, for all $i \in N$ and $\mathscr{F}=\{N\}$. It is clear that $\left\langle\mathscr{F},\left\langle\mathscr{A}_{i}, \mathscr{V}_{i}\right\rangle_{i \in N}\right\rangle$ is a representation of $\langle\mathscr{A}, \mathscr{U}\rangle$ in the noncompensatory sorting model with veto.

Suppose henceforth that some attribute is influent for $\left\langle\mathscr{A}^{\prime}, \mathscr{U}^{\prime}\right\rangle$ so that $\mathscr{A}^{\prime} \neq \varnothing$ and $\mathscr{U}^{\prime} \neq \varnothing$. The plan is to apply Theorem 21 to the partition $\left\langle\mathscr{A}^{\prime}, \mathscr{U}^{\prime}\right\rangle$ on $Y$. It is clear that $\left\langle\mathscr{A}^{\prime}, \mathscr{U}^{\prime}\right\rangle$ on $Y$ is linear. Let us show that $\left\langle\mathscr{A}^{\prime}, \mathscr{U}^{\prime}\right\rangle$ on $Y$ is 2-graded, which will complete the proof, in view of Theorem 21. Suppose, in contradiction with the thesis, that $\left(x_{i}, a_{-i}\right) \in \mathscr{A}^{\prime}$, $\left(y_{i}, a_{-i}\right) \in \mathscr{A}^{\prime}\left(y_{i}, b_{-i}\right) \in \mathscr{A}^{\prime}\left(x_{i}, b_{-i}\right) \in \mathscr{U}^{\prime}$ and $\left(z_{i}, a_{-i}\right) \in \mathscr{U}^{\prime}$. Since, by construction, $z_{i} \in Y_{i}$, we know that $\left(z_{i}, c_{-i}\right) \in \mathscr{A}^{\prime}$, for some $c_{-i} \in Y_{-i}$. Using $3 \mathrm{v}$-graded $i,\left(x_{i}, a_{-i}\right) \in \mathscr{A}^{\prime},\left(y_{i}, a_{-i}\right) \in \mathscr{A}^{\prime},\left(y_{i}, b_{-i}\right) \in \mathscr{A}^{\prime}$ and $\left(z_{i}, c_{-i}\right) \in \mathscr{A}^{\prime}$ imply $\left(x_{i}, b_{-i}\right) \in \mathscr{A}^{\prime}$ or $\left(z_{i}, a_{-i}\right) \in \mathscr{A}^{\prime}$, a contradiction.

\subsection{Uniqueness}

As in the above proof, let $Z_{i}=\left\{x_{i} \in X_{i}:\left(x_{i}, a_{-i}\right) \in \mathscr{U}\right.$, for all $\left.a_{-i} \in X_{-i}\right\}$ and $Y_{i}=X_{i} \backslash Z_{i}$. Let $Y=\prod_{i=1}^{n} Y_{i}$ and define $\mathscr{A}^{\prime}=\mathscr{A} \cap Y$ and $\mathscr{U}^{\prime}=\mathscr{U} \cap Y$. Since $\langle\mathscr{A}, \mathscr{U}\rangle$ is a partition, $Y$ is always nonempty. We claim that the representation $\left\langle\mathscr{F},\left\langle\mathscr{A}_{i}, \mathscr{V}_{i}\right\rangle_{i \in N}\right\rangle$ of $\langle\mathscr{A}, \mathscr{U}\rangle$ in the noncompensatory sorting model with veto is unique if and only if all attributes $i \in N$ are influent for $\left\langle\mathscr{A}^{\prime}, \mathscr{U}^{\prime}\right\rangle$.

Suppose that the above condition holds. Let us first show that, in all representations $\left\langle\mathscr{F},\left\langle\mathscr{A}_{i}, \mathscr{V}_{i}\right\rangle_{i \in N}\right\rangle$ of $\langle\mathscr{A}, \mathscr{U}\rangle$, we must have $\mathscr{V}_{i}=Z_{i}$. By construction, $x_{i} \in \mathscr{V}_{i}$ implies $x_{i} \in Z_{i}$. Conversely, suppose that $x_{i} \in Z_{i}$ and that $x_{i} \notin \mathscr{V}_{i}$. We must have either $x_{i} \in \mathscr{A}_{i}$ or $x_{i} \in \mathscr{U}_{i}$. Since $i$ is influent for $\left\langle\mathscr{A}^{\prime}, \mathscr{U}^{\prime}\right\rangle$ on $Y$, there are $y_{i}, z_{i} \in Y_{i}$ such that $\left(y_{i}, a_{-i}\right) \in \mathscr{A}$ and $\left(z_{i}, a_{-i}\right) \in \mathscr{U}$, for some $a_{-i} \in Y_{-i}$. This implies $y_{i} \in \mathscr{A}_{i}$ and $z_{i} \in \mathscr{U}_{i}$. Since $z_{i} \in Y_{i}$, we know that $\left(z_{i}, b_{-i}\right) \in \mathscr{A}$, for some $b_{-i} \in Y_{-i}$. If $x_{i} \in \mathscr{A}_{i},\left(y_{i}, a_{-i}\right) \in \mathscr{A}$ implies, using the definition of the noncompensatory sorting model with veto, $\left(x_{i}, a_{-i}\right) \in \mathscr{A}$, a contradiction. If $x_{i} \in \mathscr{U}_{i},\left(z_{i}, b_{-i}\right) \in \mathscr{A}$ implies $\left(x_{i}, b_{-i}\right) \in \mathscr{A}$, a contradiction. Therefore, we must have $Z_{i}=\mathscr{V}_{i}$. Since each attribute is influent for $\left\langle\mathscr{A}^{\prime}, \mathscr{U}^{\prime}\right\rangle$, we know that the representation of $\left\langle\mathscr{A}^{\prime}, \mathscr{U}^{\prime}\right\rangle$ on $Y$ in the noncompensatory sorting model is unique.

Conversely, suppose that the above condition is violated so that some attribute $j \in N$ is degenerate for $\left\langle\mathscr{A}^{\prime}, \mathscr{U}^{\prime}\right\rangle$. Define $\mathscr{V}_{i}$ as above. We know from the analysis in Section 5 that there will be several representations of $\left\langle\mathscr{A}^{\prime}, \mathscr{U}^{\prime}\right\rangle$ in the noncompensatory sorting model. Hence, the representation of $\langle\mathscr{A}, \mathscr{U}\rangle$ in the noncompensatory sorting model with veto will not be unique. This proves the claim. 
The construction of a unique representation of a partition in the noncompensatory sorting model with veto is illustrated below.

\section{Example 36}

Let $n=3$ and $X_{1}=X_{2}=X_{3}=\{8,9,10,11\}$. Let $\mathscr{A}=\{(9,10,10)$, $(9,10,11),(9,11,10),(9,11,11),(10,9,10),(10,9,11),(10,10,9),(10,10,10)$, $(10,10,11),(10,11,9),(10,11,10),(10,11,11),(11,9,10),(11,9,11),(11,10,9)$, $(11,10,10),(11,10,11),(11,11,9),(11,11,10),(11,11,11)\}$ and $\mathscr{U}=X \backslash \mathscr{A}$. The partition $\langle\mathscr{A}, \mathscr{U}\rangle$ can be obtained using the pessimistic version of ELECTRE TRI with $p=(10,10,10), S_{i}=\geq$ for all $i \in N, w_{1}=w_{2}=w_{3}=1 / 3$, $\lambda=2 / 3$, and $V_{i}=\{(10,8),(11,8)\}$ for all $i \in N$. This shows that it is linear and 3 -graded with veto.

We have $Z_{1}=Z_{2}=Z_{3}=\{8\}$ and $Y=\{9,10,11\} \times\{9,10,11\} \times$ $\{9,10,11\}$. All attributes are influent for $\left\langle\mathscr{A}^{\prime}, \mathscr{U}^{\prime}\right\rangle$ on $Y$. The unique representation of $\langle\mathscr{A}, \mathscr{U}\rangle$ in the noncompensatory sorting model with veto is such that $\mathscr{A}_{1}=\mathscr{A}_{2}=\mathscr{A}_{3}=\{10,11\}, \mathscr{V}_{1}=\mathscr{V}_{2}=\mathscr{V}_{3}=\{8\}$ and $\mathscr{F}=\{\{1,2\}$, $\{1,3\},\{2,3\},\{1,2,3\}\}$.

\section{Remark 37}

Consider a partition $\langle\mathscr{A}, \mathscr{U}\rangle$ that has a representation $\left\langle\mathscr{F},\left\langle\mathscr{A}_{i}, \mathscr{V}_{i}\right\rangle_{i \in N}\right\rangle$ in the noncompensatory sorting model with veto. It is not restrictive to suppose that this representation has been obtained following the construction in the proof of Theorem 35. Take, on all $i \in N, S_{i}=\succsim_{i}$ so that $S_{i}$ are weak orders (and, hence, semiorders). Let $Z_{i}=\left\{x_{i} \in X_{i}:\left(x_{i}, a_{-i}\right) \in \mathscr{U}\right.$, for all $a_{-i} \in$ $\left.X_{-i}\right\}, Y_{i}=X_{i} \backslash Z_{i}$ and $Y=\prod_{i=1}^{n} Y_{i}$. Define $\mathscr{A}^{\prime}=\mathscr{A} \cap Y$ and $\mathscr{U}^{\prime}=\mathscr{U} \cap Y$.

If $Z_{i}=\varnothing$, take $V_{i}=\varnothing$. If $Z_{i} \neq \varnothing$, we have $\mathscr{V}_{i}=Z_{i}$. Take $V_{i}$ to be such that all elements not in $\mathscr{V}_{i}$ bear $V_{i}$ to all elements in $\mathscr{V}_{i}$. If attribute $i \in N$ is degenerate for $\left\langle\mathscr{A}^{\prime}, \mathscr{U}^{\prime}\right\rangle$, we know that we can take $\mathscr{A}_{i}$ to be an arbitrary subset of $Y_{i}$. We take it equal to $Y_{i}$ and take $p_{i}$ to be an arbitrary element in $Y_{i}$. If attribute $i \in N$ is influent for $\left\langle\mathscr{A}^{\prime}, \mathscr{U}^{\prime}\right\rangle$, we have taken $\mathscr{A}_{i}$ to coincide with the first equivalence class of $\succsim_{i}$ on $Y_{i}$. Take $p_{i}$ to be an arbitrary element in $\mathscr{A}_{i}$.

It is clear that with such a definition, $V_{i}$ is included in $\succ_{i}$. Furthermore, it is a strict semiorder. Indeed, $x_{i} V_{i} y_{i}$ is equivalent to saying that $x_{i} \notin \mathscr{V}_{i}$ and $y_{i} \in \mathscr{V}_{i}$. Therefore $x_{i} V_{i} y_{i}$ and $z_{i} V_{i} w_{i}$ will imply both $x_{i} V_{i} w_{i}$ and $z_{i} V_{i} y_{i}$. Hence, $V_{i}$ is a Ferrers relation. The fact that $V_{i}$ is semi-transitive follows from the fact that it is never true that $x_{i} V_{i} y_{i}$ and $y_{i} V_{i} z_{i}$.

Observe that, with such definitions, we have $x_{i} S_{i} p_{i}$ iff $x_{i} \in \mathscr{A}_{i}$ and $p_{i} V_{i} x_{i}$ iff $x_{i} \in \mathscr{V}_{i}$. This implies

$$
\begin{aligned}
x \in \mathscr{A} & \Leftrightarrow\left[\left\{i \in N: x_{i} \in \mathscr{A}_{i}\right\} \in \mathscr{F} \text { and }\left\{i \in N: x_{i} \in \mathscr{V}_{i}\right\}=\varnothing\right] \\
& \Leftrightarrow\left[S(x, p) \in \mathscr{F} \text { and }\left\{i \in N: p_{i} V_{i} x_{i}\right\}=\varnothing\right]
\end{aligned}
$$


If the set $\mathscr{F}$ has a joint additive representation à la ELECTRE, i.e., that, for all $i \in N$, there are nonnegative weights $w_{i}$ and a real number $\lambda$ between $1 / 2$ and 1 such that, for all $I \subseteq 2^{N}$,

$$
I \in \mathscr{F} \Leftrightarrow \sum_{i \in I} w_{i} \geq \lambda
$$

the above construction is exactly equivalent to the concordance part of ELECTRE TRI.

Neglecting the question of the additive representation of the set $\mathscr{F}$, this shows that a twofold partition that can be obtained in ELECTRE TRI using a profile that is outside the set $X$ can always be obtained with ELECTRE TRI with a profile that belongs to $X$.

\subsection{Extensions}

Let us first observe that, since the noncompensatory sorting model with veto is a particular case of model $(D 1)$, it can be expressed in the decision rule model of Greco et al. (2001b); for all $i \in N$ such that $\mathscr{V}_{i} \neq \varnothing$, all decision rules will have to include in their premises a condition that $x_{i} \notin \mathscr{V}_{i}$ (this can easily be expressed, using $\succsim_{i}$, in the "at least" syntax of decision rules).

As was the case for the noncompensatory sorting model, it is possible to consider other conditions that, in conjunction with linearity, enable to characterize the noncompensatory sorting model with veto. We say that $\langle\mathscr{A}, \mathscr{U}\rangle$ satisfies condition $3 \mathrm{v}$-graded ${ }_{i}^{*}$ if

$$
\left.\begin{array}{c}
\left(x_{i}, a_{-i}\right) \in \mathscr{A} \\
\text { and } \\
\left(z_{i}, c_{-i}\right) \in \mathscr{A} \\
\text { and } \\
\left(y_{i}, b_{-i}\right) \in \mathscr{A}
\end{array}\right\} \Rightarrow\left\{\begin{array}{c}
\left(x_{i}, b_{-i}\right) \in \mathscr{A} \\
\text { or } \\
\left(x_{i}, c_{-i}\right) \in \mathscr{A} \\
\text { or } \\
\left(z_{i}, b_{-i}\right) \in \mathscr{A}
\end{array}\right.
$$

for all $x_{i}, y_{i}, z_{i} \in X_{i}$ and all $a_{-i}, b_{-i}, c_{i} \in X_{-i}$. $\langle\mathscr{A}, \mathscr{U}\rangle$ is said to be 3 -graded* with veto if it satisfies condition $3 v-\operatorname{graded}_{i}^{*}$ for all $i \in N$ (note that this condition is not the dual of $3 \mathrm{v}$-graded $i$ replacing $\mathscr{A}$ by $\mathscr{U}$. This is due to the fact that in the noncompensatory sorting model with veto, the role of $\mathscr{A}$ and $\mathscr{U}$ is not symmetric due to the presence of the veto).

Suppose that condition $3 \mathrm{v}$-graded ${ }_{i}^{*}$ is violated so that $\left(x_{i}, a_{-i}\right) \in \mathscr{A}$, $\left(z_{i}, c_{-i}\right) \in \mathscr{A},\left(y_{i}, b_{-i}\right) \in \mathscr{A},\left(x_{i}, b_{-i}\right) \in \mathscr{U},\left(x_{i}, c_{-i}\right) \in \mathscr{U}$ and $\left(z_{i}, b_{-i}\right) \in \mathscr{U}$. By construction, none of $x_{i}, y_{i}$ and $z_{i}$ can belong to $\mathscr{V}_{i}$. We have $\operatorname{Not}\left[x_{i} \sim_{i} y_{i}\right]$, $\operatorname{Not}\left[y_{i} \sim_{i} z_{i}\right]$ and $\operatorname{Not}\left[x_{i} \sim_{i} z_{i}\right]$, which shows that condition $3 \mathrm{v}$-graded ${ }_{i}^{*}$ is necessary for the noncompensatory sorting model with veto to hold. We have: 


\section{Lemma 38}

1. Conditions $3 \mathrm{v}$-graded $i$ and $3 \mathrm{v}$-graded ${ }_{i}^{*}$ are independent.

2. In presence of linear ${ }_{i}$, conditions $3 \mathrm{v}$-graded $i$ and $3 \mathrm{v}$-graded ${ }_{i}^{*}$ are equivalent.

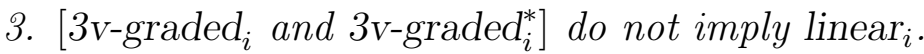

\section{ProOF}

Part 1. We give below the required two examples.

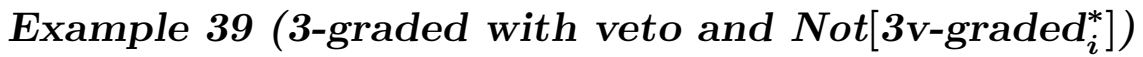

Let $n=3$ and $X=\left\{x_{1}, y_{1}, z_{1}\right\} \times\left\{x_{2}, y_{2}\right\} \times\left\{x_{3}, y_{3}\right\}$. Let $\mathscr{A}=\left\{\left(x_{1}, x_{2}, x_{3}\right)\right.$, $\left.\left(y_{1}, y_{2}, x_{3}\right),\left(z_{1}, x_{2}, y_{3}\right)\right\}$ and $\mathscr{U}=X \backslash \mathscr{A}$. Since $X_{2}$ and $X_{3}$ have only

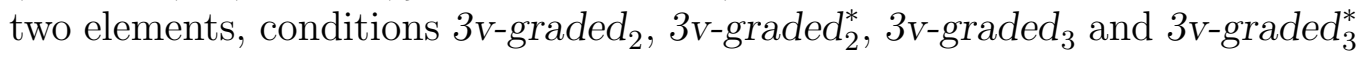
hold. Condition 3v-graded 1 is trivially satisfied. Condition $3 v$-graded ${ }_{1}^{*}$ is violated since $\left(x_{1}, x_{2}, x_{3}\right) \in \mathscr{A},\left(y_{1}, y_{2}, y_{3}\right) \in \mathscr{A}$ and $\left(z_{1}, x_{2}, y_{3}\right) \in \mathscr{A}$ but $\left(x_{1}, x_{2}, y_{3}\right) \in \mathscr{U},\left(x_{1}, y_{2}, y_{3}\right) \in \mathscr{U}$ and $\left(y_{1}, x_{2}, y_{3}\right) \in \mathscr{U}$.

Example 40 (3-graded ${ }^{*}$ with veto and $N o t\left[3 v\right.$-graded $\left.\left.{ }_{i}\right]\right)$

Let $n=3$ and $X=\left\{x_{1}, y_{1}, z_{1}\right\} \times\left\{x_{2}, y_{2}\right\} \times\left\{x_{3}, y_{3}\right\}$. Let $\mathscr{U}=\left\{\left(y_{1}, x_{2}, x_{3}\right)\right.$, $\left.\left(z_{1}, x_{2}, y_{3}\right)\right\}$ and $\mathscr{A}=X \backslash \mathscr{U}$. Since $X_{2}$ and $X_{3}$ have only two elements, conditions $3 \mathrm{v}$-graded 2 , 3v-graded 2 , $3 \mathrm{v}$-graded 3 and $3 v$-graded 3 hold. Condition $3 \mathrm{v}$-graded 1 is violated since $\left(y_{1}, x_{2}, y_{3}\right) \in \mathscr{A},\left(x_{1}, x_{2}, y_{3}\right) \in \mathscr{A},\left(x_{1}, x_{2}, x_{3}\right) \in$ $\mathscr{A}$ and $\left(z_{1}, y_{2}, x_{3}\right) \in \mathscr{A}$ but $\left(y_{1}, x_{2}, x_{3}\right) \in \mathscr{U}$ and $\left(z_{1}, x_{2}, y_{3}\right) \in \mathscr{U}$. It is routine to check that $3 \mathrm{v}$-graded $d_{1}^{*}$ is satisfied.

Part 2. Let us first show that linear $i$ and $3 v$-graded $i$ imply $3 v-$ graded $_{i}^{*}$. Suppose, contrary to the thesis, that $\left(x_{i}, a_{-i}\right) \in \mathscr{A},\left(z_{i}, c_{-i}\right) \in \mathscr{A},\left(y_{i}, b_{-i}\right) \in$ $\mathscr{A},\left(x_{i}, b_{-i}\right) \in \mathscr{U},\left(x_{i}, c_{-i}\right) \in \mathscr{U}$ and $\left(z_{i}, b_{-i}\right) \in \mathscr{U}$. Using linear $r_{i},\left(z_{i}, c_{-i}\right) \in$

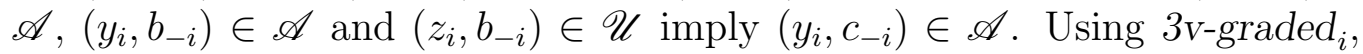
$\left(z_{i}, c_{-i}\right) \in \mathscr{A},\left(y_{i}, c_{-i}\right) \in \mathscr{A},\left(y_{i}, b_{-i}\right) \in \mathscr{A}$ and $\left(x_{i}, a_{-i}\right) \in \mathscr{A}$ imply $\left(z_{i}, b_{-i}\right) \in$ $\mathscr{A}$ or $\left(x_{i}, c_{-i}\right) \in \mathscr{A}$, a contradiction.

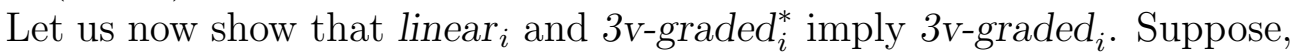
contrary to the thesis, that $\left(x_{i}, a_{-i}\right) \in \mathscr{A},\left(y_{i}, a_{-i}\right) \in \mathscr{A},\left(y_{i}, b_{-i}\right) \in \mathscr{A}$ and $\left(z_{i}, c_{-i}\right) \in \mathscr{A}$ while $\left(x_{i}, b_{-i}\right) \in \mathscr{U}$ and $\left(z_{i}, a_{-i}\right) \in \mathscr{U}$. We must have $\left(z_{i}, b_{-i}\right) \in \mathscr{U}$, otherwise, using $\operatorname{linear}_{i},\left(x_{i}, a_{-i}\right) \in \mathscr{A}$ and $\left(z_{i}, b_{-i}\right) \in \mathscr{A}$ would imply either $\left(z_{i}, a_{-i}\right) \in \mathscr{A}$ or $\left(x_{i}, b_{-i}\right) \in \mathscr{A}$. Using $3 \mathrm{v}$-graded ${ }_{i}^{*},\left(z_{i}, c_{-i}\right) \in \mathscr{A}$, $\left(x_{i}, a_{-i}\right) \in \mathscr{A}$ and $\left(y_{i}, b_{-i}\right) \in \mathscr{A}$ imply $\left(z_{i}, a_{-i}\right) \in \mathscr{A}$ or $\left(z_{i}, b_{-i}\right) \in \mathscr{A}$ or $\left(x_{i}, b_{-i}\right) \in \mathscr{A}$, a contradiction.

Part 3. When $X_{i}$ has two elements, conditions $3 v$-graded $i$ and $3 v$-graded ${ }_{i}^{*}$ are trivially satisfied. Therefore, in Example 18, conditions $3 \mathrm{v}$-graded $i$ and $3 \mathrm{v}$-graded ${ }_{i}^{*}$ are satisfied for all $i \in N$, while condition linear $_{i}$ hold on all but one attribute. 
This shows that we may replace 3 -graded with veto by 3 -graded* with veto in the characterization of the noncompensatory sorting model with veto proposed in Theorem 35. Furthermore, the conjunction of these two conditions has interesting consequences on its own. Consider a model such that:

- for all $i \in N$ there are disjoint sets $\mathscr{A}_{i}, \mathscr{N}_{i} \subseteq X_{i}$,

- there is a subset $\mathscr{F}$ of $2^{N}$,

such that, for all $x \in X$,

$$
x \in \mathscr{A} \Leftrightarrow\left[\left\{i \in N: x_{i} \in \mathscr{A}_{i}\right\} \in \mathscr{F} \text { and }\left\{i \in N: x_{i} \in \mathscr{V}_{i}\right\}=\varnothing\right] .
$$

The only difference between this model and the noncompensatory sorting model with veto is that $\mathscr{F}$ is no longer supposed to satisfy (11). Hence, in such a model, an object $x \in X$ belongs to $\mathscr{A}$ if it has a combination of elements in $\mathscr{A}_{i}$ that is typical of $\mathscr{A}$, while a repulsive evaluation for $\mathscr{A}$ is able to destroy this typicalness (e.g., being [red and sporty] implies that a car is attractive, except, e.g., if it comes from country $C$, where $C$ stands for a country having the reputation to build poor cars). This model is a particular case of model $(D 0)$ in which each function $u_{i}$ can take at most three distinct values and, when $u_{i}$ takes exactly three distinct values, the lower value of $u_{i}$ forbids to have a positive value for $F$. We call this model the generalized noncompensatory model with veto. We have:

\section{Proposition 41}

A partition $\langle\mathscr{A}, \mathscr{U}\rangle$ has a representation in the generalized noncompensatory model with veto iff it is 3-graded with veto and 3-graded* with veto.

PROOF

The necessity of $3 \mathrm{v}$-graded $i$ and $3 \mathrm{v}$-graded ${ }_{i}^{*}$ is easily shown. We show sufficiency. For all $i \in N$, let $\mathscr{V}_{i}=\left\{x_{i} \in X_{i}:\left(x_{i}, a_{-i}\right) \in \mathscr{U}\right.$, for all $\left.a_{-i} \in X_{-i}\right\}$. Let $Y_{i}=X_{i} \backslash \mathscr{V}_{i}$ and $Y=\prod_{i=1}^{n} Y_{i}$. Since $\langle\mathscr{A}, \mathscr{U}\rangle$ is a partition, $Y$ must be nonempty.

For each $i \in N$, we distinguish two cases.

1. If, for all $x_{i}, y_{i} \in Y_{i}$, we have $x_{i} \sim_{i} y_{i}$, we define $\mathscr{A}_{i}=Y_{i}$.

2. If, for some $x_{i}, y_{i} \in Y_{i}$ we have $\operatorname{Not}\left[x_{i} \sim_{i} y_{i}\right]$, so that $\left(x_{i}, a_{-i}^{*}\right) \in \mathscr{A}$ and $\left(y_{i}, a_{-i}^{*}\right) \in \mathscr{U}$, for some $a_{-i}^{*} \in Y_{-i}$, take any such $a_{-i}^{*} \in Y_{-i}$ and define $\mathscr{A}_{i}=\left\{z_{i} \in Y_{i}:\left(z_{i}, a_{-i}^{*}\right) \in \mathscr{A}\right\}$. In this case, by construction, $\mathscr{A}_{i} \neq \varnothing$ and $\mathscr{A}_{i} \neq Y_{i}$. 
Define $\mathscr{F}$ letting $I \in \mathscr{F}$ whenever there is some $x \in \mathscr{A}$ such that $A(x)=I$. Let us show that, for all $x \in Y=\prod_{i \in N} Y_{i}$, we have:

$$
x \in \mathscr{A} \Leftrightarrow A(x) \in \mathscr{F},
$$

which will complete the proof. By construction, if $x \in \mathscr{A}$, we have $A(x) \in \mathscr{F}$.

Conversely, suppose that $A(x) \in \mathscr{F}$. This implies that $A(y)=A(x)$, for some $y \in Y$ such that $y \in \mathscr{A}$.

Take any $i \in N$. Let us show that we have $\left(x_{i}, y_{-i}\right) \in \mathscr{A}$. If $\mathscr{A}_{i}=Y_{i}$, we know that $y_{i} \sim_{i} x_{i}$ and, hence, $\left(x_{i}, y_{-i}\right) \in \mathscr{A}$. Suppose that $\mathscr{A}_{i} \neq Y_{i}$. By construction, we know that:

- $\left(x_{i}, a_{-i}^{*}\right)$ and $\left(y_{i}, a_{-i}^{*}\right)$ both belong either to $\mathscr{A}$ or to $\mathscr{U}$.

- $\left(z_{i}, a_{-i}^{*}\right) \in \mathscr{A}$ and $\left(w_{i}, a_{-i}^{*}\right) \in \mathscr{U}$, for some $z_{i}, w_{i} \in Y_{i}$.

Suppose that $\left(x_{i}, a_{-i}^{*}\right) \in \mathscr{A}$ and $\left(y_{i}, a_{-i}^{*}\right) \in \mathscr{A}$. Because $w_{i} \in Y_{i}$, we know that $\left(w_{i}, b_{-i}\right) \in \mathscr{A}$, for some $b_{-i} \in Y_{-i}$. Using $3 v-\operatorname{graded}_{i},\left(x_{i}, a_{-i}^{*}\right) \in \mathscr{A}$, $\left(y_{i}, a_{-i}^{*}\right) \in \mathscr{A},\left(y_{i}, y_{-i}\right) \in \mathscr{A}$ and $\left(w_{i}, b_{-i}\right) \in \mathscr{A}$ imply, $\left(x_{i}, y_{-i}\right) \in \mathscr{A}$ or $\left(w_{i}, a_{-i}^{*}\right) \in \mathscr{A}$. Hence, we must have $\left(x_{i}, y_{-i}\right) \in \mathscr{A}$.

Suppose now that $\left(x_{i}, a_{-i}^{*}\right) \in \mathscr{U}$ and $\left(y_{i}, a_{-i}^{*}\right) \in \mathscr{U}$. Because $x_{i} \in Y_{i}$, we know that $\left(x_{i}, c_{-i}\right) \in \mathscr{A}$, for some $c_{-i} \in Y_{-i}$. Using $3 v$-graded ${ }_{i}^{*},\left(x_{i}, c_{-i}\right) \in \mathscr{A}$, $\left(y_{i}, y_{-i}\right) \in \mathscr{A}$ and $\left(z_{i}, a_{-i}^{*}\right) \in \mathscr{A}$ imply $\left(x_{i}, a_{-i}^{*}\right) \in \mathscr{A}$ or $\left(x_{i}, y_{-i}\right) \in \mathscr{A}$ or $\left(y_{i}, a_{-i}^{*}\right) \in \mathscr{A}$. Therefore, we must have $\left(x_{i}, y_{-i}\right) \in \mathscr{A}$.

In any case, we have $\left(x_{i}, y_{-i}\right) \in \mathscr{A}$. The proof is completed iterating the above reasoning (beginning with "Take any $i \in N$ ") using $\left(x_{i}, y_{-i}\right) \in \mathscr{A}$ instead of $y \in \mathscr{A}$ as a starting point and considering an attribute $j \neq i$.

We leave to the reader the, easy task, of formulating the generalized noncompensatory model with veto in terms of decision rules. Since this model is not a particular case of model $(D 1)$, this formulation may not always be done only using "at least" decision rules.

Consider a model such that:

- for all $i \in N$ there are disjoint sets $\mathscr{A}_{i}, \mathscr{V}_{i} \subseteq X_{i}$,

- there is a subset $\mathscr{F}$ of $2^{N}$,

such that, for all $x \in X$,

$$
x \in \mathscr{A} \Leftrightarrow\left[\left\{i \in N: x_{i} \in \mathscr{A}_{i}\right\} \in \mathscr{F} \text { or }\left\{i \in N: x_{i} \in \mathscr{V}_{i}\right\} \neq \varnothing\right]
$$

This model is closely related to the generalized noncompensatory model with veto. The only difference is that the elements of $\mathscr{V}_{i}$ play a dual role: instead of 
being repulsive, i.e., forbidding an alternative to belong to $\mathscr{A}$, an evaluation in $\mathscr{V}_{i}$ is now "compulsive" for $\mathscr{A}$, i.e., it forces an alternative to be in $\mathscr{A}$. Paraphrasing the proof of Proposition 41, it is possible to show that this model is characterized by two conditions obtained replacing $\mathscr{A}$ by $\mathscr{U}$ in the expression of $3 \mathrm{v}$-graded $i$ and $3 \mathrm{v}$-graded ${ }_{i}^{*}$. We leave the details to the interested reader.

\section{Discussion}

This paper has analyzed a number of sorting models for multi-attributed alternatives into two categories. The common feature of these models is to particularize model $(D 1)$ in the direction of using poor information on each attribute. Indeed, when there is no veto effect involved, noncompensatory models only distinguish two types of elements on each attribute. The possibility of veto effects adds a possible third type of elements.

The conditions that we have exhibited are reasonably simple and could well be the subject of empirical tests. A psychologist may, for instance, want to use them in order to know whether a partition of alternatives given by a subject can be explained using a noncompensatory model. On a more conceptual level, our conditions allow to pinpoint what appears to be the main distinctive feature of noncompensatory models à la ELECTRE TRI within the general framework of model $(D 1)$. This was already shown in a series of papers (see Bouyssou and Pirlot, 2002a, 2005; Dubois et al., 2003; Greco et al., 2001a) for the case of models involving binary relations. Our analysis can be considered as an extension of these papers to the case of sorting models. As a by-product of this investigation, the comments of Salvatore Greco, Benedetto Matarazzo and Roman Słowiński lead us to realize that, rather unexpectedly, the noncompensatory sorting model is, in fact, equivalent to the sorting model $(S u)$ based on the Sugeno integral characterized in Słowiński et al. (2002). This gives an alternative interpretation of model (Su) that is simple and, we feel, more attractive than the one suggested by the original formula.

Our theoretical analysis also has practical implications. First, it shows that, beyond surface, the two versions of ELECTRE TRI are rather different: only the pessimistic version fits into the framework of noncompensatory sorting models. This is related with the fact that most works trying to infer the parameters of an ELECTRE TRI model from assignment examples (i.e., from a partition defined on a subset of $X$ ) using mathematical programming techniques (see Dias and Mousseau, 2006; Dias et al., 2002; Mousseau et al., 2001a; Mousseau and Słowiński, 1998; Ngo The and Mousseau, 2002) have 
only considered the pessimistic version of the method. Indeed, our models seem to show that the optimistic version of ELECTRE TRI is at variance with the general principles underlying most of the other ELECTRE-like techniques (see Roy, 1991). Furthermore, we have shown that the conditions ensuring the uniqueness of a representation in the noncompensatory sorting model with veto are rather stringent, requiring much more than the influence of each attribute. Such a non-uniqueness will all the more be an issue for methods designed to infer all the parameters of an ELECTRE TRI model from assignment examples (see Mousseau and Słowiński, 1998) since they work on the basis of even less information than we do here. This possible, and likely, non-uniqueness of the representation probably explains why this type of method, independently of its computational complexity involving the solution of nonlinear programs, have been abandoned and replaced by techniques inferring only one type of parameter (e.g., weights, veto thresholds or category limits) at a time.

Finally, it should be mentioned that, contrary to what happens in ELECTRE TRI, the models proposed in this paper do not assume numerical weights (not to mention the possibility to add them in order to test if a coalition of attributes is judged "sufficiently" important). Indeed, as shown in Greco et al. (2001b) and Słowiński et al. (2002), these models can be formulated in terms of decision rules having a particular syntax. Hence, it is possible to use symbolic inference techniques derived from Artificial Intelligence to assess them on the basis of assignment examples (see Greco et al., 1999, 2001c, 2002b, 2005, for reviews of this line of research that also has many applications outside the area of sorting models). Therefore, this absence of weights should not be considered as an impediment to the practical use of such models.

The analysis proposed in this paper can be extended in several directions. First it is clearly necessary to extend our results concerning noncompensatory models to more than two categories. This is done in a companion paper (Bouyssou and Marchant, 2005). Although relatively straightforward, this extension raises several delicate points, so that we have decided not to include it in the present paper. Quite a different line of extension is linked with the study of additive models for sorting. Using standard techniques, such an analysis is relatively straightforward when the set of alternatives is finite; it nevertheless raises difficult questions in the general case. This is the subject of an ongoing research. Furthermore, as proposed by Goldstein (1991), sorting models in which some objects are "in between" two categories (e.g., at the border between $\mathscr{A}$ and $\mathscr{U}$ ) would deserve attention. Models using "prototypes" for each category instead of limiting profiles should also be studied; we have seen that it is not unlikely that model $(D 1)$ offers an adequate 
framework for doing so. As suggested in Greco et al. (2001b) and Słowiński et al. (2002), the study of models in which some alternatives may belong to more than one category, e.g., because of uncertainty, imprecision or lack of information, may prove useful. Finally, it should mentioned that Greco et al. (2001b) and Słowiński et al. (2002) have considered a generalization of model (D1) allowing for violations of linearity, exploiting the idea of the "rough approximation" of a partition. More generally, it seems that the use of conjoint measurement techniques applied to partitions of multi-attributed alternatives offers much promise for future research into the foundations of MCDM techniques.

\section{References}

Andenmatten, A., 1995. Évaluation du risque de défaillance des émetteurs d'obligations, une approche par l'aide multicritère à la décision. Presses Polytechniques et Universitaires Romandes, Lausanne.

Arondel, C., Girardin, P., 2000. Sorting cropping systems on the basis of their impact on groundwater quality. European Journal of Operational Research 127 (3), 467-482.

Belton, V., Stewart, T., 2001. Multiple criteria decision analysis: An integrated approach. Kluwer, Dordrecht.

Bouyssou, D., Marchant, Th., 2005. An axiomatic approach to noncompensatory sorting methods in MCDM, II: More than two categories, Working paper, LAMSADE, Université Paris Dauphine, available at www.lamsade.dauphine.fr/ $\sim$ bouyssou/.

Bouyssou, D., Marchant, Th., Pirlot, M., Perny, P., Tsoukiàs, A., Vincke, Ph., 2000. Evaluation and decision models: A critical perspective. Kluwer, Dordrecht.

Bouyssou, D., Pirlot, M., 1999. Conjoint measurement without additivity and transitivity. In: Meskens, N., Roubens, M. (Eds.), Advances in Decision Analysis. Kluwer, Dordrecht, pp. 13-29.

Bouyssou, D., Pirlot, M., 2002a. A characterization of strict concordance relations. In: Bouyssou, D., Jacquet-Lagrèze, É., Perny, P., Słowiński, R., Vanderpooten, D., Vincke, Ph. (Eds.), Aiding Decisions with Multiple Criteria: Essays in Honour of Bernard Roy. Kluwer, Dordrecht, pp. 121-145.

Bouyssou, D., Pirlot, M., 2002b. Nontransitive decomposable conjoint measurement. Journal of Mathematical Psychology 46, 677-703.

Bouyssou, D., Pirlot, M., 2004a. 'Additive difference' models without additivity and subtractivity. Journal of Mathematical Psychology 48 (4), 263-291.

Bouyssou, D., Pirlot, M., 2004b. Preferences for multiattributed alternatives: Traces, dominance, and numerical representations. Journal of Mathematical Psychology 48 (3), 167-185. 
Bouyssou, D., Pirlot, M., 2005. A characterization of concordance relations. European Journal of Operational Research 167 (2), 427-443.

Dias, L. C., Clímaco, J., 2000. ELECTRE TRI for groups with imprecise information on parameter values. Group Decision and Negotiation 9, 355-377.

Dias, L. C., Mousseau, V., 2006. Inferring ELECTRE's veto-related parameters from outranking examples. European Journal of Operational Research 170 (1), $172-191$.

Dias, L. C., Mousseau, V., Figueira, J., Clímaco, J., 2002. An aggregation / disaggregation approach to obtain robust conclusions with ELECTRE TRI. European Journal of Operational Research 138, 332-48.

Doumpos, M., Zopounidis, C., 2002. On the development of an outranking relation for ordinal classification problems: An experimental investigation of a new methodology. Optimization Methods ad Software 17, 293-317.

Doumpos, M., Zopounidis, C., 2004. A multicriteria classification approach based on pairwise comparisons. European Journal of Operational Research 158, 378389.

Dubois, D., Fargier, H., Perny, P., Prade, H., 2003. A characterization of generalized concordance rules in multicriteria decision-making. International Journal of Intelligent Systems 18 (7), 751-774.

Figueira, J., De Smet, Y., Brans, J.-P., 2004. MCDA methods for sorting and clustering problems: PROMETHEE TRI and PROMETHEE CLUSTER. Research report, SMG - ULB.

Fishburn, P. C., 1970. Utility theory for decision-making. Wiley, New York.

Fishburn, P. C., 1978. A survey of multiattribute/multicriteria evaluation theories. In: Zionts, S. (Ed.), Multicriteria problem solving. Springer-Verlag, Berlin, pp. 181-224.

Georgopoulou, E., Sarafidis, Y., Mirasgedis, S., Zaimi, S., Lalas, D., 2003. A multiple criteria decision-aid approach in defining national priorities for greenhouse gases emissions reduction in the energy sector. European Journal of Operational Research 146 (1), 199-215.

Goldstein, W. M., 1991. Decomposable threshold models. Journal of Mathematical Psychology 35, 64-79.

Greco, S., Matarazzo, B., Słowiński, R., 1999. The use of rough sets and fuzzy sets in MCDM. In: Gal, T., Hanne, T., Stewart, T. (Eds.), Multicriteria decision making, Advances in MCDM models, algorithms, theory and applications. Kluwer, Dordrecht, pp. 14.1-14.59.

Greco, S., Matarazzo, B., Słowiński, R., 2001a. Axiomatic basis of noncompensatory preferences, communication to FUR X, 30 May-2 June, Torino, Italy.

Greco, S., Matarazzo, B., Słowiński, R., 2001b. Conjoint measurement and rough set approach for multicriteria sorting problems in presence of ordinal criteria. In: Colorni, A., Paruccini, M., Roy, B. (Eds.), A-MCD-A, Aide Mulcritère à la Décision / Multiple Criteria Decision Aid. European Commission, Joint Research Centre, Luxembourg, pp. 117-144. 
Greco, S., Matarazzo, B., Słowiński, R., 2001c. Rough sets theory for multicriteria decision analysis. European Journal of Operational Research 129, 1-7.

Greco, S., Matarazzo, B., Słowiński, R., 2002a. Multicriteria classification. In: Klogsen, W., Zytkow, J. (Eds.), Handbook of data mining and knowledge discovery. Oxford University Press, Oxford, pp. 318-328.

Greco, S., Matarazzo, B., Słowiński, R., 2002b. Rough sets methodology for sorting problems in presence of multiple attributes and criteria. European Journal of Operational Research 138, 247-59.

Greco, S., Matarazzo, B., Słowiński, R., 2005. Decision rule approach. In: Figueira, J., Greco, S., Ehrgott, M. (Eds.), Multiple Criteria Decision Analysis: State of the Art Surveys. Springer Verlag, Boston, Dordrecht, London, pp. 507-562.

Hand, D. J., 1981. Discrimination and classification. Wiley, New York.

Henriet, L., 2000. Systèmes d'évaluation et de classification multicritères pour l'aide à la décision. construction de modèles et procédures d'affectation. thèse de doctorat, Université Paris Dauphine, France.

Jacquet-Lagrèze, É., 1995. An application of the UTA discriminant model for the evaluation of R\&D projects. In: Pardalos, P., Siskos, Y., Zopounidis, C. (Eds.), Advances in Multicriteria Analysis. Kluwer, Dordrecht, pp. 203-211.

Keeney, R. L., Raiffa, H., 1976. Decisions with multiple objectives: Preferences and value tradeoffs. Wiley, New York.

Köksalan, M., Ulu, C., 2003. An interactive approach for placing alternatives in preference classes. European Journal of Operational Research 144, 429-439.

Krantz, D. H., Luce, R. D., Suppes, P., Tversky, A., 1971. Foundations of measurement, vol. 1: Additive and polynomial representations. Academic Press, New York.

Lourenco, R. P., Costa, J. P., 2004. Using ELECTRE TRI outranking method to sort MOMILP non dominated solutions. European Journal of Operational Research 153 (2), 271-289.

Marichal, J.-L., 2000. On Sugeno integrals as an aggregation function. Fuzzy Sets and Systems 114 .

Marichal, J.-L., Meyer, P., Roubens, M., 2005. Sorting multi-attribute alternatives: The TOMASO method. Computers \& Operations Research 32.

Marichal, J.-L., Roubens, M., 2001. On a sorting procedure in the presence of qualitative interacting points of view. In: Chojean, J., Leski, J. (Eds.), Fuzzy Sets and their Applications. Silesian University Press, Gliwice, pp. 217-230.

Massaglia, R., Ostanello, A., 1991. N-tomic: A support system for multicriteria segmentation problems. In: Korhonen, P., Lewandowski, A., Wallenius, J. (Eds.), Multiple Criteria Decision Support. Vol. 356 of Lecture Notes in Economics and Mathematical Systems. IIASA, pp. 167-174, proceedings of the International Workshop, Helsinki.

Meyer, P., Roubens, M., 2005. Choice, ranking and sorting in fuzzy multiple criteria decision aid. In: Figueira, J., Greco, S., Ehrgott, M. (Eds.), Multiple 
Criteria Decision Analysis: State of the Art Surveys. Springer Verlag, Boston, Dordrecht, London, pp. 471-506.

Moscarola, J., Roy, B., 1977. Procédure automatique d'examen de dossiers fondée sur une segmentation trichotomique en présence de critères multiples. RAIRO/Operations Resarch 11 (2), 145-173.

Moussa, N., 2001. Aide multicritère à l'évaluation qualitative par inférence de modèles de tri ordinal sur une hiérarchie de critères. Thèse de doctorat, Université Paris-Dauphine.

Mousseau, V., Dias, L. C., 2004. Valued outranking relations in ELECTRE providing manageable disaggregation procedures. European Journal of Operational Research 156, 467-482.

Mousseau, V., Figueira, J., Naux, J.-Ph., 2001a. Using assignment examples to infer weights for ELECTRE TRI method: Some experimental results. European Journal of Operational Research 130, 263-275.

Mousseau, V., Roy, B., Sommerlatt, I., 2000a. Elaboration d'un outil d'aide à la décision en vue de l'évolution de la tarification des transports publics en Ile de France. Journal of Decision Systems 9 (2), 289-315.

Mousseau, V., Roy, B., Sommerlatt, I., 2001b. Development of a decision aiding tool for the evolution of public transport ticket pricing in the Paris region. In: A. Colorni, M. P., Roy, B. (Eds.), A-MCD-A Aide Multicritère à la Décision - Multiple Criteria Decision Aiding. European Commission, Joint Research Centre, Luxembourg, pp. 213-230.

Mousseau, V., Słowiński, R., 1998. Inferring an ELECTRE TRI model from assignment examples. Journal of Global Optimization 12, 157-174.

Mousseau, V., Słowiński, R., Zielniewicz, P., 2000b. A user-oriented implementation of the ELECTRE TRI method integrating preference elicitation support. Computers \& Operations Research 27, 757-777.

Nakamura, Y., 2004. Trichotomic preferences for gambles. Journal of Mathematical Psychology 48 (6), 385-398.

Ngo The, A., Mousseau, V., 2002. Using assignment examples to infer category limits for the ELECTRE TRI method. Journal of Multi-Criteria Decision Analysis 11 (1), 29-43.

Norese, M., Viale, S., 2002. A multi-profile sorting procedure in the public administration. European Journal of Operational Research 138, 365-79.

Pawlak, Z., 1991. Rough Sets. Theoretical aspects of reasoning about data. Kluwer, Dordrecht.

Pawlak, Z., Słowiński, R., 1994. Decision analysis using rough sets. International Transactions on Operational Research 1, 107-114.

Perny, P., 1998. Multicriteria filtering methods based on concordance and nondiscordance principles. Annals of Operations Research 80, 137-165.

Roy, B., 1981. A multicriteria analysis for trichotomic segmentation problems. In: Nijkamp, P., Spronk, J. (Eds.), Multiple Criteria Analysis: Operational methods. Gower Publishing Company, Aldershot, England, pp. 245-257. 
Roy, B., 1991. The outranking approach and the foundations of ELECTRE methods. Theory and Decision 31, 49-73.

Roy, B., 1996. Multicriteria methodology for decision aiding. Kluwer, Dordrecht, original version in French: "Méthodologie multicritère d'aide à la décision", Economica, Paris, 1985.

Roy, B., March 2002. Présentation et interprétation de la méthode ELECTRE TRI pour affecter des zones dans des catégories de risque. Document du LAMSADE 124, Université de Paris Dauphine, 25 pages.

Roy, B., Bouyssou, D., 1993. Aide multicritère à la décision : méthodes et cas. Economica, Paris.

Słowiński, R., Greco, S., Matarazzo, B., 2002. Axiomatization of utility, outranking and decision-rule preference models for multiple-criteria classification problems under partial inconsistency with the dominance principle. Control and Cybernetics 31 (4), 1005-1035.

Tervonen, T., Almeida-Dias, J., Figueira, J., Lahdelma, R., Salminen, P., 2005. SMAA-TRI: A parameter stability analysis method for ELECTRE TRI. Research Report 6, INESC - Coimbra.

Wakker, P. P., 1989. Additive representations of preferences: A new foundation of decision analysis. Kluwer, Dordrecht.

Wei, Y., 1992. Aide multicritère à la décision dans le cadre de la problématique du tri : concepts, méthodes et applications. Thèse de doctorat, Université Paris Dauphine, Paris.

Weiss, S. M., Kulikowski, C. A., 1991. Computer Systems that Learn: Classification and prediction methods from statistics, neural nets, machine learning and experts systems. Morgan Kaufmann, San Mateo, CA.

von Winterfeldt, D., Edwards, W., 1986. Decision analysis and behavioral research. Cambridge University Press, Cambridge.

Zopounidis, C., Doumpos, M., 2000a. Building additive utilities for multi-group hierarchical discrimination: The MHDIS method. Optimization Methods \& Software 14 (3), 219-240.

Zopounidis, C., Doumpos, M., 2000b. Intelligent decision aiding systems based on multiple criteria for financial engineering. Kluwer, Dordrecht.

Zopounidis, C., Doumpos, M., 2000c. PREFDIS: A multicriteria decision support system for sorting decision problems. Computers \& Operations Research 27 (78), 779-797.

Zopounidis, C., Doumpos, M., 2002. Multicriteria classification and sorting methods: A literature review. European Journal of Operational Research 138, 229246 . 